\title{
ON THE EXISTENCE OF INTEGER RELATIVE HEFFTER ARRAYS
}

\author{
FIORENZA MORINI AND MARCO ANTONIO PELLEGRINI
}

\begin{abstract}
Let $v=2 m s+t$ be a positive integer, where $t$ divides $2 m s$, and let $J$ be the subgroup of order $t$ of the cyclic group $\mathbb{Z}_{v}$. An integer Heffter array $\mathrm{H}_{t}(m, n ; s, k)$ over $\mathbb{Z}_{v}$ relative to $J$ is an $m \times n$ partially filled array with elements in $\mathbb{Z}_{v}$ such that: (a) each row contains $s$ filled cells and each column contains $k$ filled cells; (b) for every $x \in \mathbb{Z}_{v} \backslash J$, either $x$ or $-x$ appears in the array; (c) the elements in every row and column, viewed as integers in $\pm\left\{1, \ldots,\left\lfloor\frac{v}{2}\right\rfloor\right\}$, sum to 0 in $\mathbb{Z}$.

In this paper we study the existence of an integer $\mathrm{H}_{t}(m, n ; s, k)$ when $s$ and $k$ are both even, proving the following results. Suppose that $4 \leq s \leq n$ and $4 \leq k \leq m$ are such that $m s=n k$. Let $t$ be a divisor of $2 m s$. (a) If $s, k \equiv 0(\bmod 4)$, there exists an integer $\mathrm{H}_{t}(m, n ; s, k)$. (b) If $s \equiv 2(\bmod 4)$ and $k \equiv 0(\bmod 4)$, there exists an integer $\mathrm{H}_{t}(m, n ; s, k)$ if and only if $m$ is even. (c) If $s \equiv 0(\bmod 4)$ and $k \equiv 2(\bmod 4)$, then there exists an integer $\mathrm{H}_{t}(m, n ; s, k)$ if and only if $n$ is even. (d) Suppose that $m$ and $n$ are both even. If $s, k \equiv 2(\bmod 4)$, then there exists an integer $\mathrm{H}_{t}(m, n ; s, k)$.
\end{abstract}

\section{INTRODUCTION}

Relative Heffter arrays are partially filled arrays (p.f. arrays for short) introduced in [12], generalizing the original idea of Dan Archdeacon, [1. They are defined as follows.

Definition 1.1. Let $v=2 m s+t$ be a positive integer, where $t$ divides $2 m s$, and let $J$ be the subgroup of order $t$ of the cyclic group $\mathbb{Z}_{v}$. $\mathrm{A}_{t}(m, n ; s, k)$ Heffter array over $\mathbb{Z}_{v}$ relative to $J$ is an $m \times n$ p.f. array with elements in $\mathbb{Z}_{v}$ such that:

(a) each row contains $s$ filled cells and each column contains $k$ filled cells;

(b) for every $x \in \mathbb{Z}_{v} \backslash J$, either $x$ or $-x$ appears in the array;

(c) the elements in every row and column sum to 0 .

In the square case (i.e., when $m=n$ and so $s=k$ ), the array $\mathrm{H}_{t}(n, n ; k, k)$ will be denoted by $\mathrm{H}_{t}(n ; k)$. Note that when $t=1$, that is $J$ is the trivial subgroup of $\mathbb{Z}_{2 m s+1}$, one retrieves the classical concept of Heffter array. 'Classical' Heffter arrays have been studied in several papers, mainly because they allow to produce biembeddings of orthogonal cyclic cycle decompositions of the complete graph $K_{v}$ on $v$ vertices onto orientable surfaces (see [6, 8, 11, 14] and [9]). Analogously, exploiting their connection with relative difference families (see [12] and [4, 5, 16]), relative Heffter arrays can be used for constructing pairs of orthogonal cyclic decompositions (one decomposition consisting of $s$-cycles and the other one consisting of $k$ cycles) of the complete multipartite graph $K_{\ell \times t}$ with $\ell=\frac{v}{t}$ parts, each of size $t$. Also, under suitable conditions (in particular, one needs orderings of the cells that satisfy certain properties), one can obtain biembeddings of these pairs of orthogonal cyclic decompositions of $K_{\ell \times t}$ onto orientable surfaces (see [13]).

2010 Mathematics Subject Classification. 05B20; 05B30.

Key words and phrases. Relative Heffter array; multipartite complete graph; cyclic decomposition. 
A relative Heffter array is called integer if Condition (c) in Definition 1.1 is strengthened so that the elements in every row and in every column, viewed as integers in $\pm\left\{1, \ldots,\left\lfloor\frac{v}{2}\right\rfloor\right\}$, sum to zero in $\mathbb{Z}$. The support of an integer Heffter array $A$, denoted by $\operatorname{supp}(A)$, is defined to be the set of the absolute values of the elements contained in $A$. It easily follows that an integer $\mathrm{H}_{2}(m, n ; s, k)$ is nothing but an integer $\mathrm{H}_{1}(m, n ; s, k)$, since in both cases the support is the set $\{1,2, \ldots, m s\}$.

The existence problem for square Heffter arrays $\mathrm{H}_{1}(n ; k)$ has been considered and solved in a series of recent papers. In particular, by [3, 15] integer Heffter arrays $\mathrm{H}_{1}(n ; k)$ (and integer $\left.\mathrm{H}_{2}(n ; k)\right)$ exist if and only if $n \geq k \geq 3$ and $n k \equiv 0,3(\bmod 4)$. Dropping the integer assumption, in [7] it was proved that Heffter arrays $\mathrm{H}_{1}(n ; k)$ exist for all $n \geq k \geq 3$. Regarding non-square arrays, only the tight case has been solved: in [2] it was proved that there exists a $\mathrm{H}_{1}(m, n ; n, m)$ for all $m, n \geq 3$, and there exists an integer $\mathrm{H}_{1}(m, n ; n, m)$ if and only if the additional condition $m n \equiv 0,3(\bmod 4)$ holds.

Necessary conditions for the existence of an integer $\mathrm{H}_{t}(m, n ; s, k)$, in addition to the trivial ones $3 \leq s \leq n, 3 \leq k \leq m$ and $m s=n k$, are given by the following result which can be easily deduced from [12, Proposition 3.1], reapplying the original argument on the columns.

Proposition 1.2. Suppose there exists an integer $\mathrm{H}_{t}(m, n ; s, k)$ for some divisor $t$ of $2 m s=$ $2 n k$.

(1) If $t$ divides $m s$, then $m s \equiv 0(\bmod 4)$ or $m s \equiv-t \equiv \pm 1(\bmod 4)$.

(2) If $t=2 m s$, then $s$ and $k$ must be both even.

(3) If $t \neq 2 m s$ does not divide $m s$, then $t+2 m s \equiv 0(\bmod 8)$.

The previous results on integer $\mathrm{H}_{1}(n ; k)$ state that for $t=1,2$ these necessary conditions are actually also sufficient. The same holds also for $t=k \neq 5$, as proved in [12] (the existence of an integer $\mathrm{H}_{5}(n ; 5)$ is still an open problem for $\left.n \equiv 0(\bmod 4)\right)$. However, in the same paper the authors showed that there is no integer $\mathrm{H}_{3 n}(n ; 3)$ and no integer $\mathrm{H}_{8}(4 ; 3)$, even if conditions of Proposition 1.2 hold. The existence of an integer $\mathrm{H}_{n}(n ; 3)$ and of an integer $\mathrm{H}_{2 n}(n ; 3)$ was proved in 13 for all odd $n \geq 3$. No other case has been studied so far: this leaves the existence problem of an integer $\mathrm{H}_{t}(m, n ; s, k)$ widely open.

In this paper we consider the case when $s$ and $k$ are both even. In particular, we show that the previous necessary conditions are also sufficient when $s$ and $k$ are even and $m s \equiv 0$ $(\bmod 4)$. In other words, we prove the following result.

Theorem 1.3. Let $m, n, s, k$ be integers such that $4 \leq s \leq n, 4 \leq k \leq m$ and $m s=n k$. Let $t$ be a divisor of $2 \mathrm{~ms}$.

(1) If $s, k \equiv 0(\bmod 4)$, then there exists an integer $\mathrm{H}_{t}(m, n ; s, k)$.

(2) If $s \equiv 2(\bmod 4)$ and $k \equiv 0(\bmod 4)$, then there exists an integer $\mathrm{H}_{t}(m, n ; s, k)$ if and only if $m$ is even.

(3) If $s \equiv 0(\bmod 4)$ and $k \equiv 2(\bmod 4)$, then there exists an integer $\mathrm{H}_{t}(m, n ; s, k)$ if and only if $n$ is even.

(4) Suppose that $m$ and $n$ are both even. If $s, k \equiv 2(\bmod 4)$, then there exists an integer $\mathrm{H}_{t}(m, n ; s, k)$.

This result is proved constructively in Sections 3, 4and 5 item (1) follows from Proposition 3.5 items (2) and (3) follow from Proposition 4.11 item (4) follows from Proposition 5.2. Unfortunately, the case when $m$ and $n$ are both odd and $s, k \equiv 2(\bmod 4)$ remains open. Note that, under these hypotheses, $t$ cannot be a divisor of $m s$. 
To conclude, we point out that from Theorem 1.3, [12, Proposition 2.9] and [10, Theorem 4.1] we obtain the following result concerning cyclic cycle decompositions of $\frac{K_{2 m s+t}}{t} \times t$.

Corollary 1.4. Let $m, n, s, k$ be positive such that $4 \leq s \leq n, 4 \leq k \leq m$ and $m s=n k$. Let $t$ be a divisor of $2 \mathrm{~ms}$. There exists a pair $\left(\mathcal{D}_{1}, \mathcal{D}_{2}\right)$ of orthogonal cyclic decompositions of the graph $K_{\frac{2 m s+t}{t} \times t}$, where $\mathcal{D}_{1}$ consists of s-cycles and $\mathcal{D}_{2}$ consists of $k$-cycles, in each of the following cases:

(1) $(s, k) \in\{(4,4),(4,6),(4,8),(6,4),(6,8),(8,4),(8,6),(8,8)\}$;

(2) $(s, k)=(6,6)$, with $m$ and $n$ both even.

This result can be extended to any even $s$ and $k$ assuming the validity of [10, Conjecture 3].

\section{ACKNOWLEDGMENTS}

The second author was supported by the National Group for Algebraic and Geometric Structures, and their Applications (GNSAGA-INdAM).

\section{Notations}

In this paper, the arithmetic on the row (respectively, on the column) indices is performed modulo $m$ (respectively, modulo $n$ ), where the set of reduced residues is $\{1,2, \ldots, m\}$ (respectively, $\{1,2, \ldots, n\}$ ), while the entries of the arrays are taken in $\mathbb{Z}$. Given two integers $a \leq b$, we denote by $[a, b]$ the interval containing the integers $\{a, a+1, \ldots, b\}$. If $a>b$, then $[a, b]$ is empty. We denote by $(i, j)$ the cell in the $i$-th row and $j$-th column of an array $A$. The skeleton of $A$ is the set of its filled positions.

If $A$ is an $m \times n$ p.f. array, for $i \in[1, n]$ we define the $i$-th diagonal as

$$
D_{i}=\{(1, i),(2, i+1), \ldots,(m, i+m-1)\} .
$$

Definition 2.1. A p.f. array with entries in $\mathbb{Z}$ is said to be shiftable if every row and every column contains an equal number of positive and negative entries.

Let $A$ be a shiftable p.f. array and $x$ be a nonnegative integer. Let $A \pm x$ be the (shiftable) p.f. array obtained adding $x$ to each positive entry of $A$ and $-x$ to each negative entry of $A$. Observe that, since $A$ is shiftable, the row and column sums of $A \pm x$ are exactly the row and column sums of $A$.

Given a sequence $S=\left(B_{1}, B_{2}, \ldots\right)$ of shiftable p.f. arrays and a nonnegative integer $x$, we write $S \pm x$ for the sequence $\left(B_{1} \pm x, B_{2} \pm x, \ldots\right)$.

We denote by $\tau_{i}(A)$ and $\gamma_{j}(A)$ the sum of the elements of the $i$-th row and the sum of the elements of the $j$-th column, respectively, of a p.f. array $A$.

If $S_{1}=\left(a_{1}, a_{2}, \ldots, a_{r}\right)$ and $S_{2}=\left(b_{1}, b_{2}, \ldots, b_{u}\right)$ are two sequences, by $S_{1}+S_{2}$ we mean the sequence $\left(a_{1}, a_{2}, \ldots, a_{r}, b_{1}, b_{2}, \ldots, b_{u}\right)$ obtained by concatenation of $S_{1}$ and $S_{2}$. In particular, if $S_{1}$ is the empty sequence then $S_{1}+S_{2}=S_{2}$. Furthermore, given the sequences $S_{1}, \ldots, S_{c}$, we write $\underset{i=1}{\stackrel{c}{\#}} S_{i}$ for $\left(\cdots\left(\left(S_{1}+S_{2}\right)+S_{3}\right)+\cdots\right)+S_{c}$.

Finally, we recall that if $A$ is an integer $\mathrm{H}_{t}(m, n ; s, k)$, then

$$
\left.\operatorname{supp}(A)=\left[1, m s+\left\lfloor\frac{t}{2}\right\rfloor\right\rfloor \backslash \ell, 2 \ell, \ldots,\left\lfloor\frac{t}{2}\right\rfloor \ell\right\}, \quad \text { where } \ell=\frac{2 m s}{t}+1 .
$$




\section{THE CASE $s, k \equiv 0(\bmod 4)$}

In this section we prove the existence of an integer $\mathrm{H}_{t}(m, n ; s, k)$ when both $s$ and $k$ are divisible by 4 . First of all, we set

$$
d=\operatorname{gcd}(m, n), \quad m=d \bar{m}, \quad n=d \bar{n}, \quad s=4 \bar{s} \quad \text { and } \quad k=4 \bar{k} .
$$

Note that from $m s=n k$ we obtain that $\bar{n}$ divides $\bar{s}$ and $\bar{m}$ divides $\bar{k}$. Hence, we can write $\bar{s}=c \bar{n}$ and $\bar{k}=c \bar{m}$.

Fix two integers $a, b \geq 2$ and consider the following shiftable p.f. array:

$$
B=B_{a, b}=\begin{array}{|c|c|}
\hline 1 & -(a+1) \\
\hline & \\
\hline-(b+1) & a+b+1 \\
\hline
\end{array}
$$

Note that the sequences of the row/column sums are $(-a, a)$ and $(-b, b)$, respectively. We use this $3 \times 2$ block for constructing p.f. arrays whose rows and columns sum to zero. Start taking an empty $m \times n$ array $A$, fix a set $X$ of $m \bar{n}$ nonnegative integers $x_{0}, x_{1}, \ldots, x_{m \bar{n}-1}$, and arrange the blocks $B \pm x_{j}$ in such a way that the element $1+x_{j}$ fills the cell $(j+1, j+1)$ of $A$ (recall that we work modulo $m$ on row indices and modulo $n$ on column indices). In this way, we fill the diagonals $D_{i m-1}, D_{i m}, D_{i m+1}, D_{i m+2}$ with $i \in[1, \bar{n}]$. In particular, every row has $4 \bar{n}$ filled cells and every column has $4 \bar{m}$ filled cells.

Looking at the rows, the elements belonging to the diagonals $D_{i m+1}, D_{i m+2}$ sum to $-a$, while the elements belonging to the diagonals $D_{i m-1}, D_{i m}$ sum to $a$. Looking at the columns, the elements belonging to the diagonals $D_{i m+1}, D_{i m-1}$ sum to $-b$, while the elements belonging to the diagonals $D_{i m+2}, D_{i m}$ sum to $b$. Then $A$ has row/column sums equal to zero.

Applying this process $c$ times (working with the diagonals $D_{i m+3}, D_{i m+4}, D_{i m+5}, D_{i m+6}$, and so on), we obtain a p.f. array $A$, whose rows have exactly $4 \bar{n} \cdot c=s$ filled cells and whose columns have exactly $4 \bar{m} \cdot c=k$ filled cells.

Example 3.1. For $a=2$ and $b=5$, fixing $X=\{0,1,10,11,20,21,30,31,40,41,50,51\}$, we can fill the diagonals $D_{1}, D_{2}, D_{5}, D_{6}, D_{7}, D_{8}, D_{11}, D_{12}$ of the following $6 \times 12$ p.f. array, where we highlighted the block $B_{2,5}$ :

$A=$\begin{tabular}{|c|c|c|c|c|c|c|c|c|c|c|c|}
\hline 1 & -3 & & & -26 & 28 & 31 & -33 & & & -56 & 58 \\
\hline 59 & 2 & -4 & & & -27 & 29 & 32 & -34 & & & -57 \\
\hline-6 & 8 & 11 & -13 & & & -36 & 38 & 41 & -43 & & \\
\hline & -7 & 9 & 12 & -14 & & & -37 & 39 & 42 & -44 & \\
\hline & & -16 & 18 & 21 & -23 & & & -46 & 48 & 51 & -53 \\
\hline-54 & & & -17 & 19 & 22 & -24 & & & -47 & 49 & 52 \\
\hline
\end{tabular}

Note that $\operatorname{supp}(A)=[1,60] \backslash\{5 j: j \in[1,12]\}$. As the reader can verify, $A$ is an integer $\mathrm{H}_{24}(6,12 ; 8,4)$ : in this case $\ell=\frac{2 \cdot 6 \cdot 8}{24}+1=5$.

The three constructions we present in this section are obtained following this procedure, so they all produce p.f. arrays of size $m \times n$ whose rows and columns sum to zero. To obtain an integer $\mathrm{H}_{t}(m, n ; s, k)$ with $s, k \equiv 0(\bmod 4)$, we only have to determine two integers $a, b \geq 2$ and a set $X=\left\{x_{0}, x_{1}, \ldots, x_{m s / 4-1}\right\} \subset \mathbb{N}$ such that the p.f. array constructed using the blocks $B_{a, b} \pm x_{j}$ has the right support. For instance, we can arrange the blocks in such a way that the element $1+x_{j}$ fills the cell $\left(j+1,4 q_{j}+j+1\right)$, where $q_{j}$ is the quotient of the division of $j$ by $\operatorname{lcm}(m, n)$. 
Throughout this section we always assume that $4 \leq s \leq n, 4 \leq k \leq m, m s=n k$ and $s, k \equiv 0(\bmod 4)$.

Lemma 3.2. There exists an integer $\mathrm{H}_{t}(m, n ; s, k)$ for any divisor $t$ of $2 m s$ such that $t \equiv 0$ $(\bmod 8)$.

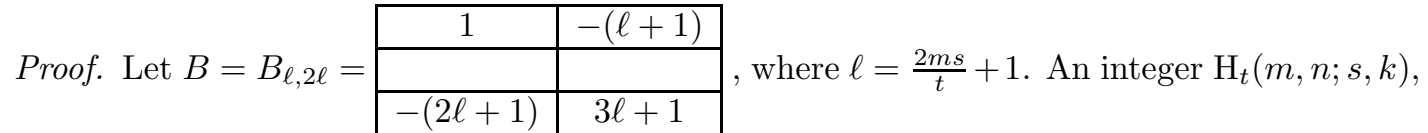

say $A$, can be obtained following the construction described before, once we exhibit a suitable set $X$ of size $\frac{m s}{4}$, in such a way that $\operatorname{supp}(A)=\left[1, m s+\frac{t}{2}\right] \backslash\left\{\ell, 2 \ell, \ldots, \frac{t}{2} \ell\right\}$.

Start considering the set $X_{0}=[0, \ell-2]$ of size $\ell-1=\frac{2 m s}{t}$ : it is easy to see that $\bigcup_{x \in X_{0}} \operatorname{supp}(B \pm x)=[1,4 \ell] \backslash\{\ell, 2 \ell, 3 \ell, 4 \ell\}$. Similarly, for any $i \in \mathbb{N}$, if $X_{i}=[4 i \ell,(4 i+1) \ell-2]$, then

$$
\bigcup_{x \in X_{i}} \operatorname{supp}(B \pm x)=[4 i \ell+1,(4 i+4) \ell] \backslash\{(4 i+1) \ell,(4 i+2) \ell,(4 i+3) \ell,(4 i+4) \ell\} .
$$

Clearly, $X_{i_{1}} \cap X_{i_{2}}=\emptyset$ if $i_{1} \neq i_{2}$. So, take $X=\bigcup_{i=0}^{t / 8-1} X_{i}$ : this is a set of size $\frac{t}{8} \cdot \frac{2 m s}{t}=\frac{m s}{4}$, as required. Also, the p.f. array $A$ obtained using the blocks $B \pm x$ with $x \in X$ has support equal to

$$
\begin{aligned}
\operatorname{supp}(A) & =\bigcup_{i=0}^{t / 8-1}([4 i \ell+1,(4 i+4) \ell] \backslash\{(4 i+1) \ell,(4 i+2) \ell,(4 i+3) \ell,(4 i+4) \ell\}) \\
& =\left[1, \frac{t}{2} \ell\right] \backslash\left\{\ell, 2 \ell, \ldots, \frac{t}{2} \ell\right\}=\left[1, m s+\frac{t}{2}\right] \backslash\left\{\ell, 2 \ell, \ldots, \frac{t}{2} \ell\right\} .
\end{aligned}
$$

This shows that $A$ is an integer $\mathrm{H}_{t}(m, n ; s, k)$.

For instance, to construct an integer $\mathrm{H}_{16}(5,10 ; 8,4)$ we can follow the proof of the previous lemma. In fact, $t=16$ divides $2 \cdot 5 \cdot 8$; note that $\ell=6$.

$\mathrm{H}_{16}(5,10 ; 8,4)=$\begin{tabular}{|c|c|c|c|c|c|c|c|c|c|}
\hline 1 & -7 & & -16 & 22 & 25 & -31 & & -40 & 46 \\
\hline 47 & 2 & -8 & & -17 & 23 & 26 & -32 & & -41 \\
\hline-13 & 19 & 3 & -9 & & -37 & 43 & 27 & -33 & \\
\hline & -14 & 20 & 4 & -10 & & -38 & 44 & 28 & -34 \\
\hline-35 & & -15 & 21 & 5 & -11 & & -39 & 45 & 29 \\
\hline
\end{tabular}

Lemma 3.3. There exists an integer $\mathrm{H}_{t}(m, n ; s, k)$ for any divisor $t$ of $m s$ such that $t \equiv 0$ $(\bmod 4)$.

Proof. Let $B=B_{1, \ell}=$\begin{tabular}{|c|c|}
\hline 1 & -2 \\
\hline & \\
\hline
\end{tabular} and note that, since $t$ divides $m s, \ell=\frac{2 m s}{t}+1$ is an odd integer. We start considering the set $X_{0}=\{0,2,4, \ldots, \ell-3\}$ of size $\frac{\ell-1}{2}=\frac{m s}{t}$ : it is easy to see that $\bigcup_{x \in X_{0}} \operatorname{supp}(B \pm x)=[1, \ell-1] \cup[\ell+1,2 \ell-1]=[1,2 \ell] \backslash\{\ell, 2 \ell\}$. Similarly, for any $i \in \mathbb{N}$, if $X_{i}=\{2 i \ell, 2 i \ell+2,2 i \ell+4, \ldots,(2 i+1) \ell-3\}$, then

$$
\bigcup_{x \in X_{i}} \operatorname{supp}(B \pm x)=[2 i \ell+1,2(i+1) \ell] \backslash\{(2 i+1) \ell,(2 i+2) \ell\}
$$


and $X_{i_{1}} \cap X_{i_{2}}=\emptyset$ if $i_{1} \neq i_{2}$. So, take $X=\bigcup_{i=0}^{t / 4-1} X_{i}$ : this is a set of size $\frac{t}{4} \cdot \frac{m s}{t}=\frac{m s}{4}$, as required. Hence, the p.f. array $A$ obtained following our procedure and using the blocks $B \pm x$ with $x \in X$ has support equal to

$$
\begin{aligned}
\operatorname{supp}(A) & =\bigcup_{i=0}^{t / 4-1}([2 i \ell+1,2(i+1) \ell] \backslash\{(2 i+1) \ell,(2 i+2) \ell\}) \\
& =\left[1, \frac{t}{2} \ell\right] \backslash\left\{\ell, 2 \ell, \ldots, \frac{t}{2} \ell\right\}=\left[1, m s+\frac{t}{2}\right] \backslash\left\{\ell, 2 \ell, \ldots, \frac{t}{2} \ell\right\} .
\end{aligned}
$$

It follows that $A$ is an integer $\mathrm{H}_{t}(m, n ; s, k)$.

Following the proof of the previous lemma, we can construct an integer $\mathrm{H}_{12}(9 ; 8)$. In fact, $t=12$ divides $9 \cdot 8$; note that $\ell=13$.

$\mathrm{H}_{12}(9 ; 8)=$\begin{tabular}{|c|c|c|c|c|c|c|c|c|}
\hline 1 & -2 & -74 & 75 & 33 & -34 & & -42 & 43 \\
\hline 45 & 3 & -4 & -76 & 77 & 35 & -36 & & -44 \\
\hline-14 & 15 & 5 & -6 & -46 & 47 & 37 & -38 & \\
\hline & -16 & 17 & 7 & -8 & -48 & 49 & 53 & -54 \\
\hline-56 & & -18 & 19 & 9 & -10 & -50 & 51 & 55 \\
\hline 57 & -58 & & -20 & 21 & 11 & -12 & -66 & 67 \\
\hline 69 & 59 & -60 & & -22 & 23 & 27 & -28 & -68 \\
\hline-70 & 71 & 61 & -62 & & -24 & 25 & 29 & -30 \\
\hline-32 & -72 & 73 & 63 & -64 & & -40 & 41 & 31 \\
\hline
\end{tabular}

Lemma 3.4. There exists an integer $\mathrm{H}_{t}(m, n ; s, k)$ for any divisor $t$ of $\frac{m s}{2}$.

Proof. Let $B=B_{1,2}=$\begin{tabular}{|c|c|}
\hline 1 & -2 \\
\hline & \\
\hline-3 & 4 \\
\hline
\end{tabular} . Note that $\ell=\frac{2 m s}{t}+1 \equiv 1(\bmod 4)$ since $t$ divides $\frac{m s}{2}$.

We start considering the set $X_{0}=\{0,4,8, \ldots, \ell-5\}$ of size $\frac{\ell-1}{4}=\frac{m s}{2 t}$ : it is easy to see that $\bigcup_{x \in X_{0}} \operatorname{supp}(B \pm x)=[1, \ell] \backslash\{\ell\}$. Similarly, for any $i \in \mathbb{N}$, if $X_{i}=\{i \ell, i \ell+4, i \ell+8, \ldots,(i+1) \ell-5\}$, then

$$
\bigcup_{x \in X_{i}} \operatorname{supp}(B \pm x)=[i \ell+1,(i+1) \ell] \backslash\{(i+1) \ell\}
$$

and $X_{i_{1}} \cap X_{i_{2}}=\emptyset$ if $i_{1} \neq i_{2}$.

If $t$ is even, take $X=\bigcup_{i=0}^{t / 2-1} X_{i}$ : this is a set of size $\frac{t}{2} \cdot \frac{m s}{2 t}=\frac{m s}{4}$, as required. The p.f. array $A$ obtained following our procedure and using the blocks $B \pm x$ with $x \in X$ has support equal to

$$
\begin{aligned}
\operatorname{supp}(A) & =\bigcup_{i=0}^{t / 2-1}([i \ell+1,(i+1) \ell] \backslash\{(i+1) \ell\}) \\
& =\left[1, \frac{t}{2} \ell\right] \backslash\left\{\ell, 2 \ell, \ldots, \frac{t}{2} \ell\right\}=\left[1, m s+\frac{t}{2}\right] \backslash\left\{\ell, 2 \ell, \ldots, \frac{t}{2} \ell\right\} .
\end{aligned}
$$

Suppose now that $t$ is odd. Notice that, in this case, $\ell \equiv 1(\bmod 8)$. Take

$$
Y=\left\{\left(\frac{t-1}{2}\right) \ell,\left(\frac{t-1}{2}\right) \ell+4,\left(\frac{t-1}{2}\right) \ell+8, \ldots,\left(\frac{t-1}{2}\right) \ell+\frac{\ell-9}{2}\right\} .
$$


Then $|Y|=\frac{m s}{4 t}$ and $\bigcup_{y \in Y} \operatorname{supp}(B \pm y)=\left[\left(\frac{t-1}{2}\right) \ell+1,\left(\frac{t-1}{2}\right) \ell+\frac{\ell-1}{2}\right]$. Take $X=\left(\bigcup_{i=0}^{(t-3) / 2} X_{i}\right) \cup$ $Y$ : this is a set of size $\frac{t-1}{2} \cdot \frac{m s}{2 t}+\frac{m s}{4 t}=\frac{m s}{4}$, as required. In this case, the p.f. array $A$ obtained following our procedure and using the blocks $B \pm x$ with $x \in X$ has support:

$$
\begin{aligned}
\operatorname{supp}(A) & =\bigcup_{i=0}^{\frac{t-3}{2}}([i \ell+1,(i+1) \ell] \backslash\{(i+1) \ell\}) \cup\left(\left[\left(\frac{t-1}{2}\right) \ell+1,\left(\frac{t-1}{2}\right) \ell+\frac{\ell-1}{2}\right]\right) \\
& =\left(\left[1, \frac{t-1}{2} \ell\right] \backslash\left\{\ell, 2 \ell, \ldots, \frac{t-1}{2} \ell\right\}\right) \cup\left[\left(\frac{t-1}{2}\right) \ell+1, m s+\frac{t-1}{2}\right] \\
& =\left[1, m s+\left\lfloor\frac{t}{2}\right\rfloor\right] \backslash\left\{\ell, 2 \ell, \ldots,\left\lfloor\frac{t}{2}\right\rfloor \ell\right\} .
\end{aligned}
$$

In both cases, we obtain that $A$ is an integer $\mathrm{H}_{t}(m, n ; s, k)$.

For instance, we can follow the proof of the previous lemma for constructing an integer $\mathrm{H}_{10}(5,10 ; 8,4)$. In fact, $t=10$ divides $\frac{5 \cdot 8}{2}=20$. Note that $\ell=9$.

$\mathrm{H}_{10}(5,10 ; 8,4)=$\begin{tabular}{|c|c|c|c|c|c|c|c|c|c|}
\hline 1 & -2 & & -16 & 17 & 23 & -24 & & -39 & 40 \\
\hline 44 & 5 & -6 & & -21 & 22 & 28 & -29 & & -43 \\
\hline-3 & 4 & 10 & -11 & & -25 & 26 & 32 & -33 & \\
\hline & -7 & 8 & 14 & -15 & & -30 & 31 & 37 & -38 \\
\hline-42 & & -12 & 13 & 19 & -20 & & -34 & 35 & 41 \\
\hline
\end{tabular}

Proposition 3.5. Suppose $4 \leq s \leq n, 4 \leq k \leq m, m s=n k$ and $s, k \equiv 0(\bmod 4)$. Then, there exists a shiftable integer $\mathrm{H}_{t}(m, n ; s, k)$ for every divisor $t$ of $2 m s$.

Proof. Let $t$ be a divisor of $2 m s$. If $t \equiv 0(\bmod 8)$, then we apply Lemma 3.2 . If $t \equiv 4$ $(\bmod 8)$, then $t$ divides $m s$ and hence we can apply Lemma 3.3 . Finally, if $t \not \equiv 0(\bmod 4)$, then $t$ divides $\frac{m s}{2}$ and so the existence of an integer $\mathrm{H}_{t}(m, n ; s, k)$ follows from Lemma 3.4 . Note that in all these three cases, the integer relative Heffter array that we construct is shiftable.

\section{THE CASE $s \equiv 2(\bmod 4)$ AND $k \equiv 0(\bmod 4)$}

In this section, we will assume $s \geq 6$ and $k \geq 4$ with $s \equiv 2(\bmod 4)$ and $k \equiv 0(\bmod 4)$. From $m s=n k$ it follows that $m$ must be even. To prove the existence of an integer $\mathrm{H}_{t}(m, n ; s, k)$, we start determining the skeleton of such arrays. To this purpose, we define a 'base unit' that we will fill with the elements of suitable blocks.

Let $\mathcal{B}$ be a sequence of blocks such that the following property is satisfied:

fixed $b$ integers $\sigma_{1}, \ldots, \sigma_{b}$, the elements of $\mathcal{B}$ are blocks $B$ of size $2 \times 2 b$ such that $\gamma_{2 i-1}(B)=-\gamma_{2 i}(B)=\sigma_{i}$ for all $i \in[1, b]$.

For instance, the following block satisfies condition (4.1) with $\sigma_{1}=-3, \sigma_{2}=-2$ and $\sigma_{3}=1$ :

\begin{tabular}{|r|r|r|r|r|r|}
\hline 1 & -2 & 6 & -7 & -10 & 12 \\
\hline-4 & 5 & -8 & 9 & 11 & -13 \\
\hline
\end{tabular}

So, fix two integers $a$ and $d$ such that $1 \leq 2 a \leq d$. Let $\mathcal{B}=\left(B_{1}, \ldots, B_{d}\right)$ be a sequence satisfying (4.1), where the blocks $B_{r}=\left(b_{i, j}^{(r)}\right)$ are all of size $2 \times 2 a$. Let $P=P(\mathcal{B})$ be the p.f. array of size $2 d \times d$ so defined. For all $i \in[1, a]$ and all $j \in[1,2 a]$, the cell $(i, i+j-1)$ of $P$ is filled with the element $b_{1, j}^{(i)}$ and the cell $(d+i, i+j-1)$ is filled with the element $b_{2, j}^{(i)}$; here, 
the column indices are taken modulo $d$. The remaining cells of $P$ are empty. An example of such construction is given in Figure 1.

\begin{tabular}{|c|c|c|c|c|c|}
\hline$b_{1,1}^{(1)}$ & $b_{1,2}^{(1)}$ & $b_{1,3}^{(1)}$ & $b_{1,4}^{(1)}$ & & \\
\hline & $b_{1,1}^{(2)}$ & $b_{1,2}^{(2)}$ & $b_{1,3}^{(2)}$ & $b_{1,4}^{(2)}$ & \\
\hline & & $b_{1,1}^{(3)}$ & $b_{1,2}^{(3)}$ & $b_{1,3}^{(3)}$ & $b_{1,4}^{(3)}$ \\
\hline$b_{1,4}^{(4)}$ & & & $b_{1,1}^{(4)}$ & $b_{1,2}^{(4)}$ & $b_{1,3}^{(4)}$ \\
\hline$b_{1,3}^{(5)}$ & $b_{1,4}^{(5)}$ & & & $b_{1,1}^{(5)}$ & $b_{1,2}^{(5)}$ \\
\hline$b_{1,2}^{(6)}$ & $b_{1,3}^{(6)}$ & $b_{1,4}^{(6)}$ & & & $b_{1,1}^{(6)}$ \\
\hline$b_{2,1}^{(1)}$ & $b_{2,2}^{(1)}$ & $b_{2,3}^{(1)}$ & $b_{2,4}^{(1)}$ & & \\
\hline & $b_{2,1}^{(2)}$ & $b_{2,2}^{(2)}$ & $b_{2,3}^{(2)}$ & $b_{2,4}^{(2)}$ & \\
\hline & & $b_{2,1}^{(3)}$ & $b_{2,2}^{(3)}$ & $b_{2,3}^{(3)}$ & $b_{2,4}^{(3)}$ \\
\hline$b_{2,4}^{(4)}$ & & & $b_{2,1}^{(4)}$ & $b_{2,2}^{(4)}$ & $b_{2,3}^{(4)}$ \\
\hline$b_{2,3}^{(5)}$ & $b_{2,4}^{(5)}$ & & & $b_{2,1}^{(5)}$ & $b_{2,2}^{(5)}$ \\
\hline$b_{2,2}^{(6)}$ & $b_{2,3}^{(6)}$ & $b_{2,4}^{(6)}$ & & & $b_{2,1}^{(6)}$ \\
\hline
\end{tabular}

Figure 1 . This is a $P\left(B_{1}, \ldots, B_{6}\right)$, where $B_{1}, \ldots, B_{6}$ are arrays of size $2 \times 4$.

We prove that $P$ is a p.f. array whose columns all sum to zero. Observe that every row of $P$ contains exactly $2 a$ filled cells and every column contains exactly $4 a$ elements. The elements of the $i$-th column of $P$ are

$$
b_{1,1}^{(i)}, b_{1,2}^{(i-1)}, \ldots, b_{1,2 a}^{(i+1-2 a)}, b_{2,1}^{(i)}, b_{2,2}^{(i-1)}, \ldots, b_{2,2 a}^{(i+1-2 a)},
$$

where the exponents must be read modulo $d$, with residues in $[1, d]$. Since the sequence $\mathcal{B}$ satisfies (4.1), we obtain

$$
\gamma_{i}(P)=\sum_{j=1}^{2 a} \gamma_{j}\left(B_{i+1-j}\right)=\sum_{j=1}^{2 a} \gamma_{j}\left(B_{i}\right)=\sum_{u=1}^{a}\left(\sigma_{u}-\sigma_{u}\right)=0 .
$$

Furthermore, notice that $\tau_{j}(P)=\tau_{1}\left(B_{j}\right)$ and $\tau_{d+j}(P)=\tau_{2}\left(B_{j}\right)$ for all $j \in[1, d]$.

Our strategy consists of two steps: first of all, we will show that there are suitable sequences of blocks satisfying condition (4.1); then, we will use these sequences to obtain an integer $\mathrm{H}_{t}(m, n ; s ; k)$ using p.f. arrays of type $P(\mathcal{B})$.

We actually construct sequences $\mathcal{B}$ satisfying this stronger condition:

fixed $b$ integers $\sigma_{1}, \ldots, \sigma_{b}$, the elements of $\mathcal{B}$ are shiftable blocks $B$ of size

$2 \times 2 b$ such that $\tau_{1}(B)=\tau_{2}(B)=0$ and $\gamma_{2 i-1}(B)=-\gamma_{2 i}(B)=\sigma_{i}$ for all $i \in[1, b]$.

This condition includes the former (4.1) and will help us to control the row sums.

We start considering the case when $\ell=\frac{2 m s}{t}+1$ is even.

Lemma 4.1. Let $m$ and $s$ be even integers with $m \geq 2$ and $s \geq 6$ such that there exists an odd prime $p$ dividing $s$. Let $t$ be a divisor of $2 \mathrm{~ms}$ such that $t \equiv 0(\bmod 8 p)$. There exists a sequence $\mathcal{B}$ of $\frac{m}{2}$ shiftable blocks of size $2 \times s$ such that $\mathcal{B}$ satisfies condition (4.2) and $\operatorname{supp}(\mathcal{B})=[1, m s+t / 2] \backslash\{j \ell: j \in[1, t / 2]\}$. 
Proof. Take the blocks of Figure 2, Then $W_{4}$ and $W_{6}$ satisfy property (4.2) with column sums $(-2 \ell, 2 \ell, 2 \ell,-2 \ell)$ and $(-2 \ell, 2 \ell,-2 \ell, 2 \ell, \ell,-\ell)$, respectively. Furthermore,

$$
\operatorname{supp}\left(W_{4}\right)=\{j \ell+1: j \in[0,7]\} \quad \text { and } \operatorname{supp}\left(W_{6}\right)=\{j \ell+1: j \in[0,11]\} .
$$

Let $V$ be the following $2 \times 2 p$ block:

$$
\begin{array}{l|l|l|l|l|}
\hline W_{6} & W_{4} \pm 12 \ell & W_{4} \pm 20 \ell & \cdots & W_{4} \pm(4 p-8) \ell \\
\hline
\end{array}
$$

Clearly, also $V$ satisfies (4.2) and its $\operatorname{support}$ is $\operatorname{supp}(V)=\{j \ell+1: j \in[0,4 p-1]\}$. We can use this block $V$ for constructing our sequence $\mathcal{B}$ : the $2 \times s$ blocks of $\mathcal{B}$ are obtained simply by juxtaposing $h=\frac{s}{2 p}$ blocks of type $V \pm x$, for $x \in X \subset \mathbb{N}$, following the natural order of $(X, \leq)$. So, we are left to exhibit a suitable set $X$ of size $\frac{m h}{2}$ such that the support of the corresponding sequence $\mathcal{B}$ is $[1, m s+t / 2] \backslash\{j \ell: j \in[1, t / 2]\}$.

Let first $X_{0}=[0, \ell-2]$. Then $\operatorname{supp}\left(V \pm x_{i_{1}}\right) \cap \operatorname{supp}\left(V \pm x_{i_{2}}\right)=\emptyset$ for each $x_{i_{1}}, x_{i_{2}} \in X_{0}$ such that $x_{i_{1}} \neq x_{i_{2}}$. Furthermore,

$$
\bigcup_{x \in X_{0}} \operatorname{supp}(V \pm x)=[1,4 p \ell] \backslash\{j \ell: j \in[1,4 p]\} .
$$

Similarly, for any $i \in \mathbb{N}$, if $X_{i}=[4 p i \ell,(4 p i+1) \ell-2]$ then

$$
\bigcup_{x \in X_{i}} \operatorname{supp}(V \pm x)=[1+4 p i \ell, 4 p \ell+4 p i \ell] \backslash\{j \ell: j \in[1+4 p i, 4 p+4 p i]\} .
$$

Clearly, $X_{i_{1}} \cap X_{i_{2}}=\emptyset$ if $i_{1} \neq i_{2}$. Therefore, take $X=\bigcup_{i=0}^{\frac{t}{8 p}-1} X_{i}$ : this is a set of size $\frac{t}{8 p} \cdot(\ell-1)=$ $\frac{t}{8 p} \cdot \frac{4 m p h}{t}=\frac{m h}{2}$. It follows that the sequence $\mathcal{B}$ obtained, as previously described, using the

\begin{tabular}{|c|c|c|c|c|c|c|}
\hline \multirow{2}{*}{$W_{4}=$} & 1 & $-(\ell+1)$ & $-(4 \ell+1)$ & $5 \ell+1$ & & \\
\hline & $-(2 \ell+1)$ & $3 \ell+1$ & $6 \ell+1$ & $-(7 \ell+1)$ & & \\
\hline & 1 & $\overline{-(\ell+1)}$ & $4 \ell+1$ & $-(5 \ell+1)$ & $-(8 \ell+1)$ & $10 \ell+1$ \\
\hline & $\overline{-(2 \ell+1)}$ & $3 \ell+1$ & $-(6 \ell+1)$ & $7 \ell+1$ & $9 \ell+1$ & $-(11 \ell+1)$ \\
\hline
\end{tabular}
blocks $V \pm x$, with $x \in X$, has support equal to

$$
\begin{aligned}
\operatorname{supp}(\mathcal{B}) & =\bigcup_{i=0}^{\frac{t}{8 p}-1}([1+4 p i \ell, 4 p \ell+4 p i \ell] \backslash\{j \ell: j \in[1+4 p i, 4 p+4 p i]\}) \\
& =\left[1, \frac{t}{2} \ell\right] \backslash\left\{j \ell: j \in\left[1, \frac{t}{2}\right]\right\}=\left[1, m s+\frac{t}{2}\right] \backslash\left\{\ell, 2 \ell, \ldots, \frac{t}{2} \ell\right\},
\end{aligned}
$$

as required.

\begin{tabular}{|c|c|c|c|c|c|c|c|c|c|c|c|}
\hline & \multirow{2}{*}{$=$} & 1 & -5 & 17 & -21 & -33 & 41 & 49 & -53 & -65 & 69 \\
\hline & & -9 & 13 & -25 & 29 & 37 & -45 & -57 & 61 & 73 & -77 \\
\hline \multirow{2}{*}{$B$} & & 2 & -6 & 18 & -22 & -34 & 42 & 50 & -54 & -66 & 70 \\
\hline & & -10 & 14 & -26 & 30 & 38 & -46 & -58 & 62 & 74 & -78 \\
\hline
\end{tabular}

Figure 2. Shiftable blocks satisfying condition (4.2).

Example 4.2. Suppose $m=12, s=10$ and $t=80$. Following the proof of Lemma 4.1, where $p=5, h=1, \ell=4$ and $X=\{0,1,2\} \cup\{80,81,82\}$, we obtain the following sequence $\left(B_{1}, \ldots, B_{6}\right)$ of shiftable blocks: 


\begin{tabular}{|c|c|c|c|c|c|c|c|c|c|c|c|c|}
\hline \multirow{2}{*}{$B_{3}$} & \multirow{2}{*}{$=$} & 3 & -7 & 19 & -23 & -35 & & 51 & -55 & -67 & 71 & \\
\hline & & -11 & 15 & -27 & 31 & 39 & - & -59 & 63 & 75 & -79 & \\
\hline \multirow{2}{*}{$B_{4}$} & \multirow{2}{*}{$=$} & 81 & -85 & 97 & \multicolumn{2}{|c|}{-101} & -113 & 121 & 129 & -133 & -145 & 149 \\
\hline & & -89 & 93 & -105 & 10 & & 117 & -125 & -137 & 141 & 153 & -157 \\
\hline \multirow{2}{*}{$B_{5}$} & \multirow{2}{*}{$=$} & 82 & -86 & 98 & \multicolumn{2}{|c|}{-102} & $\overline{-114}$ & 122 & 130 & -134 & -146 & 150 \\
\hline & & -90 & 94 & -106 & \multicolumn{2}{|c|}{110} & 118 & -126 & -138 & 142 & 154 & -158 \\
\hline \multirow{2}{*}{$B_{6}$} & \multirow{2}{*}{$=$} & $\overline{83}$ & -87 & $\overline{99}$ & \multicolumn{2}{|c|}{-103} & -115 & 123 & 131 & -135 & -147 & $\overline{151}$ \\
\hline & & -91 & 95 & -107 & \multicolumn{2}{|c|}{111} & 119 & -127 & $\begin{array}{l}-139 \\
\end{array}$ & 143 & 155 & -159 \\
\hline
\end{tabular}

Lemma 4.3. Let $m$ and $s$ be even integers with $m \geq 2$ and $s \geq 6$ such that there exists an odd prime $p$ dividing $s$. Let $t$ be a divisor of $\frac{2 m s}{p}$ such that $t \equiv 0(\bmod 8)$ and set $\ell=\frac{2 m s}{t}+1$. There exists a sequence $\mathcal{B}$ of $\frac{m}{2}$ blocks of size $2 \times s$ such that $\mathcal{B}$ satisfies condition (4.2) and $\operatorname{supp}(\mathcal{B})=[1, m s+t / 2] \backslash\{j \ell: j \in[1, t / 2]\}$.

Proof. By hypothesis we can write $\ell=p y+1$. The blocks $W_{4}$ and $W_{6}$ of Figure 3 satisfy property (4.2) with column sums $(-y, y, y,-y)$ and $(-2(p y+1), 2(p y+1),-2(p y+1), 2(p y+$ $1), p y+1,-(p y+1))$, respectively. Furthermore,

$$
\begin{aligned}
& \operatorname{supp}\left(W_{4}\right)=\{(j p+1) y+j+1,(j p+2) y+j+1: j \in[0,3]\}, \\
& \operatorname{supp}\left(W_{6}\right)=\{j p y+j+1,(j p+1) y+j+1,(j p+2) y+j+1: j \in[0,3]\} .
\end{aligned}
$$

Let $V$ be the following $2 \times 2 p$ block:

$$
\begin{array}{l|l|l|l|l|}
\hline W_{6} & W_{4} \pm 2 y & W_{4} \pm 4 y & \cdots & W_{4} \pm(p-3) y \\
\hline
\end{array}
$$

Clearly, also $V$ satisfies (4.2) and its support is

$$
\begin{aligned}
\operatorname{supp}(V) & =\{i y+1,(p+i) y+2,(2 p+i) y+3,(3 p+i) y+4: i \in[0, p-1]\} \\
& =\{i y+1, \ell+(i y+1), 2 \ell+(i y+1), 3 \ell+(i y+1): i \in[0, p-1]\} .
\end{aligned}
$$

We can use this block $V$ for constructing our sequence $\mathcal{B}$ as done in Lemma 4.1 it suffices to exhibit a suitable set $X$ of size $\frac{m h}{2}$, where $h=\frac{s}{2 p}$, such that the support of the corresponding sequence $\mathcal{B}$ is $[1, m s+t / 2] \backslash\{j \ell: j \in[1, t / 2]\}$.

Let first $X_{0}=[0, y-1]$. Then $\operatorname{supp}\left(V \pm x_{i_{1}}\right) \cap \operatorname{supp}\left(V \pm x_{i_{2}}\right)=\emptyset$ for each $x_{i_{1}}, x_{i_{2}} \in X_{0}$ such that $x_{i_{1}} \neq x_{i_{2}}$. Furthermore,

$$
\bigcup_{x \in X_{0}} \operatorname{supp}(V \pm x)=\bigcup_{i=0}^{3}[i \ell+1, i \ell+p y]=[1,4 \ell] \backslash\{\ell, 2 \ell, 3 \ell, 4 \ell\}
$$

Similarly, for any $i \in \mathbb{N}$, if $X_{i}=[4 i \ell, 4 i \ell+y-1]$ then

$$
\bigcup_{x \in X_{i}} \operatorname{supp}(V \pm x)=[1+4 i \ell,(4 i+4) \ell] \backslash\{(4 i+1) \ell,(4 i+2) \ell,(4 i+3) \ell,(4 i+4) \ell\} .
$$

Clearly, $X_{i_{1}} \cap X_{i_{2}}=\emptyset$ if $i_{1} \neq i_{2}$. Therefore, take $X=\bigcup_{i=0}^{\frac{t}{8}-1} X_{i}$ : this is a set of size $\frac{t}{8} \cdot y=$ $\frac{t}{8} \cdot \frac{\ell-1}{p}=\frac{t}{8} \cdot \frac{4 m h}{t}=\frac{m h}{2}$. It follows that the sequence $\mathcal{B}$ obtained using the blocks $V \pm x$, with $x \in X$, has support equal to

$$
\begin{aligned}
\operatorname{supp}(\mathcal{B}) & =\bigcup_{i=0}^{\frac{t}{8}-1}([1+4 i \ell, 4 \ell(i+1)] \backslash\{(4 i+1) \ell,(4 i+2) \ell,(4 i+3) \ell,(4 i+4) \ell\}) \\
& =\left[1, \frac{t}{2} \ell\right] \backslash\left\{\ell, 2 \ell, \ldots, \frac{t}{2} \ell\right\}=\left[1, m s+\frac{t}{2}\right] \backslash\left\{\ell, 2 \ell, \ldots, \frac{t}{2} \ell\right\}
\end{aligned}
$$

as required. 


$$
\begin{aligned}
& W_{4}=\begin{array}{|c|c|c|c|}
\hline y+1 & -((p+1) y+2) & -((2 p+1) y+3) & (3 p+1) y+4 \\
\hline-(2 y+1) & (p+2) y+2 & (2 p+2) y+3 & -((3 p+2) y+4) \\
\hline
\end{array} \\
& W^{\prime}=\begin{array}{|c|c|c|c|}
\hline 1 & -(p y+2) & y+1 & -((p+1) y+2) \\
\hline-(2 p y+3) & 3 p y+4 & -((2 p+1) y+3) & (3 p+1) y+4 \\
\hline
\end{array}, \\
& W^{\prime \prime}=\begin{array}{|c|c|}
\hline-(2 y+1) & (2 p+2) y+3) \\
\hline(p+2) y+2 & -((3 p+2) y+4) \\
\hline
\end{array} \\
& W_{6}=\begin{array}{|l|l|}
\hline W^{\prime} & W^{\prime \prime} \\
\hline
\end{array}
\end{aligned}
$$

Figure 3. Shiftable blocks $W_{4}, W_{6}$ satisfying condition (4.2).

\begin{tabular}{|c|c|c|c|c|c|c|c|c|c|c|c|c|c|}
\hline \multirow{2}{*}{$B_{1}$} & \multirow{2}{*}{$=$} & 1 & -17 & 4 & -20 & -7 & 39 & \multicolumn{2}{|c|}{10} & -26 & -42 & 58 & \\
\hline & & -33 & 49 & -36 & 52 & 23 & -55 & \multicolumn{2}{|c|}{-13} & 29 & \multicolumn{2}{|c|}{45} & \\
\hline \multirow{2}{*}{$B_{2}$} & \multirow{2}{*}{$=$} & 2 & -18 & 5 & -21 & -8 & 40 & \multicolumn{2}{|c|}{11} & -27 & -43 & \multicolumn{2}{|c|}{59} \\
\hline & & -34 & 50 & -37 & 53 & 24 & -56 & \multicolumn{2}{|c|}{-14} & 30 & \multicolumn{2}{|c|}{46} & -62 \\
\hline \multirow{2}{*}{$B_{3}$} & \multirow{2}{*}{$=$} & 3 & -19 & 6 & -22 & -9 & 41 & \multicolumn{2}{|c|}{12} & -28 & -44 & \multicolumn{2}{|c|}{60} \\
\hline & & -35 & 51 & -38 & 54 & 25 & -57 & \multicolumn{2}{|c|}{-15} & 31 & 47 & \multicolumn{2}{|c|}{-63} \\
\hline \multirow{2}{*}{$B_{4}$} & \multirow[b]{2}{*}{$=$} & 65 & -81 & 68 & -84 & -71 & \multicolumn{2}{|c|}{103} & 74 & \multicolumn{2}{|c|}{-90} & -106 & 122 \\
\hline & & -97 & 113 & -100 & 116 & 87 & \multicolumn{2}{|c|}{-119} & -77 & \multicolumn{2}{|c|}{93} & 109 & -125 \\
\hline \multirow{2}{*}{$B_{5}$} & - & 66 & -82 & 69 & -85 & -72 & 10 & & 75 & & 91 & -107 & 123 \\
\hline & - & -98 & 114 & -101 & 117 & 88 & -1 & & -78 & & 4 & 110 & -126 \\
\hline$B_{6}$ & $=$ & 67 & -83 & 70 & -86 & -73 & $\overline{11}$ & & 76 & & 92 & -108 & 124 \\
\hline$D_{6}$ & - & -99 & 115 & -102 & 118 & 89 & -1 & & -79 & & 5 & 111 & -127 \\
\hline
\end{tabular}

Example 4.4. Suppose $m=12, s=10$ and $t=16$. Following the proof of Lemma4.3, where $p=5, \ell=16, h=1, y=3$ and $X=\{0,1,2\} \cup\{64,65,66\}$, we obtain the following sequence $\left(B_{1}, \ldots, B_{6}\right)$ of shiftable blocks:

We now consider the case when $\ell=\frac{2 m s}{t}+1$ is odd (that is, $t$ divides $m s$ ). We need to distinguish three possibilities, depending on the residue class of $s$ modulo 6 . So, write $s=6 q+r$, where $q \geq 0$ and $r \in\{0,8,10\}$. We start with the following auxiliary lemma, that allows us to apply an inductive process.

Lemma 4.5. Let $h \geq 1$ and let $\rho \geq 3$ be an odd integer. There exists a sequence $\mathcal{A}=\mathcal{A}_{h, \rho}$ consisting of blocks of size $2 \times 6$ such that

(1) $\mathcal{A}$ has cardinality $h$ and satisfies condition (4.2);

(2) $\operatorname{supp}(\mathcal{A})=\left[1,12 h+\left\lfloor\frac{12 h}{\rho-1}\right\rfloor\right] \backslash\left\{\rho, 2 \rho, \ldots,\left\lfloor\frac{12 h}{\rho-1}\right\rfloor \rho\right\}$.

Proof. Consider the $2 \times 6$ shiftable blocks given in Figure 4 . Observe that $\operatorname{supp}\left(F_{3}\right)=[1,18] \backslash$ $\{3,6,9,12,15,18\}, \operatorname{supp}\left(F_{5}\right)=[1,15] \backslash\{5,10,15\}$ and $\operatorname{supp}\left(V_{j}\right)=[1,13] \backslash\{j\}$. Furthermore, each of these blocks has rows that sum to zero and columns with the following sums:

$$
F_{3}:(-1,1,-3,3,6,-6), \quad F_{5}, V_{j}=(-2,2,-2,2,1,-1) .
$$

We construct our sequence $\mathcal{A}$ distinguishing three cases: we use only blocks of type $F_{3} \pm x$; or we use only blocks of type $F_{5} \pm x$; or we use blocks of type $V_{j} \pm x$ for several choices of $j$ in the same sequence.

In several cases, we provide a basic sequence $S$ of blocks having disjoint supports. To simplify the notation, if $S=\left(B_{1}, B_{2}, \ldots, B_{r}\right)$ we write $\operatorname{supp}(S)$ instead of $\bigcup_{i=1}^{r} \operatorname{supp}\left(B_{i}\right)$. Also, 


\begin{tabular}{|c|c|c|c|c|c|c|c|c|c|c|c|c|c|c|c|}
\hline \multirow{3}{*}{$F_{3}$} & \multirow{3}{*}{$=$} & & & & & & & \multirow{3}{*}{$F_{5}$} & \multirow{3}{*}{$=$} & & & & & & \\
\hline & & 1 & -4 & -10 & 16 & 14 & -17 & & & 1 & -2 & 6 & -7 & -11 & 13 \\
\hline & & -2 & 5 & 7 & -13 & -8 & 11 & & & -3 & 4 & -8 & 9 & 12 & -14 \\
\hline \multirow{2}{*}{$V_{1}$} & \multirow{2}{*}{$=$} & 2 & -3 & 6 & -7 & -10 & 12 & \multirow{2}{*}{$V_{3}$} & \multirow{2}{*}{$=$} & 4 & 7 & -13 & 12 & -8 & -2 \\
\hline & & -4 & 5 & -8 & 9 & 11 & -13 & & & -6 & -5 & 11 & -10 & 9 & 1 \\
\hline \multirow{2}{*}{$V_{5}$} & \multirow{2}{*}{$=$} & 1 & $\overline{-2-2}$ & 6 & -7 & -10 & 12 & \multirow{2}{*}{$V_{7}$} & \multirow[b]{2}{*}{$=$} & 1 & -2 & 8 & 11 & -5 & -13 \\
\hline & & -3 & 4 & -8 & 9 & 11 & -13 & & & -3 & 4 & -10 & -9 & 6 & 12 \\
\hline \multirow{2}{*}{$V_{9}$} & \multirow{2}{*}{$=$} & 1 & -2 & 5 & -6 & -10 & 12 & \multirow{2}{*}{$V_{11}$} & \multirow{2}{*}{$=$} & 1 & -2 & -10 & $\overline{-7}$ & 13 & 5 \\
\hline & & -3 & 4 & $\begin{array}{l}-7 \\
\end{array}$ & 8 & 11 & -13 & & & -3 & 4 & 8 & 9 & \begin{tabular}{|l|}
-12 \\
\end{tabular} & -6 \\
\hline \multirow{2}{*}{$V_{13}$} & \multirow{2}{*}{$=$} & 1 & -2 & 5 & -6 & -9 & 11 & & & & & & & & \\
\hline & & -3 & 4 & -7 & 8 & 10 & -12 & & & & & & & & \\
\hline
\end{tabular}

Figure 4. Shiftable blocks of size $2 \times 6$ satisfying condition (4.2).

recall that $S \pm x$ means the sequence $\left(B_{1} \pm x, \ldots, B_{r} \pm x\right)$. It will be very useful to define the following sequence. For all $b \geq 1$ let

$$
U(b)=\left(V_{13}, V_{13} \pm 12, V_{13} \pm 24, \ldots, V_{13} \pm 12(b-1)\right) .
$$

Also, we set $U(0)$ to be the empty sequence: so, for all $b \geq 0$ the sequence $U(b)$ contains $b$ elements and $\operatorname{supp}(U(b))=[1,12 b]$.

If $\rho=3$ we take $\mathcal{A}=\underset{c=0}{\mathrm{H}-1}\left(F_{3} \pm 18 c\right)$; if $\rho=5$ we take $\mathcal{A}=\underset{c=0}{h-1}\left(F_{5} \pm 15 c\right)$.

If $\rho=12 x+7$, we take $S=\left(U(x), V_{7} \pm 12 x, U(x) \pm(12 x+13)\right)$, whence $\operatorname{supp}(S)=$ $[1,2 \rho] \backslash\{\rho, 2 \rho\}$. In this case we define $\mathcal{A}$ as the sequence of the first $h$ elements of $\underset{c=0}{+}(S \pm 2 \rho c)$.

If $\rho=12 x+9$, we take

$$
S=\left(U(x), V_{9} \pm 12 x, U(x) \pm(12 x+13), V_{5} \pm(24 x+13), U(x) \pm(24 x+26)\right) .
$$

In fact, $\operatorname{supp}(S)=[1,3 \rho] \backslash\{\rho, 2 \rho, 3 \rho\}$. In this case we define $\mathcal{A}$ as the sequence of the first $h$ elements of $\underset{c=0}{\lfloor h /|S|\rfloor}(S \pm 3 \rho c)$.

If $\rho=12 x+11$, we take

$$
\begin{aligned}
S= & \left(U(x), V_{11} \pm 12 x, U(x) \pm(12 x+13), V_{9} \pm(24 x+13), U(x) \pm(24 x+26),\right. \\
& V_{7} \pm(36 x+26), U(x) \pm(36 x+39), V_{5} \pm(48 x+39), U(x) \pm(48 x+52), \\
& \left.V_{3} \pm(60 x+52), U(x) \pm(60 x+65)\right) .
\end{aligned}
$$

We obtain $\operatorname{supp}(S)=[1,6 \rho] \backslash\{j \rho: j \in[1,6]\}$, and then $\mathcal{A}$ is the sequence consisting of the first $h$ elements of $\underset{c=0}{\stackrel{\lfloor h /|S|\rfloor}{\#}}(S \pm 6 \rho c)$.

If $\rho=12 x+13$, we take $S=\left(U(x), V_{13} \pm 12 x\right)$, whence $\operatorname{supp}(S)=[1, \rho] \backslash\{\rho\}$. In this case we define $\mathcal{A}$ as the sequence of the first $h$ elements of $\underset{c=0}{+}(S /|S|\rfloor \mid \rho c)$.

$$
\begin{aligned}
\text { If } \rho=12 x+15, \text { we take } \\
\qquad \begin{aligned}
S= & \left(U(x+1), V_{3} \pm 12(x+1), U(x) \pm(12 x+25), V_{5} \pm(24 x+25), U(x) \pm(24 x+38),\right. \\
& V_{7} \pm(36 x+38), U(x) \pm(36 x+51), V_{9} \pm(48 x+51), U(x) \pm(48 x+64), \\
& \left.V_{11} \pm(60 x+64), U(x+1) \pm(60 x+77)\right)
\end{aligned}
\end{aligned}
$$


We get $\operatorname{supp}(S)=[1,6 \rho] \backslash\{j \rho: j \in[1,6]\}$. In this case we define $\mathcal{A}$ as the sequence of the first $h$ elements of $\underset{c=0}{\stackrel{\lfloor h /|S|\rfloor}{+}}(S \pm 6 \rho c)$.

Finally, if $\rho=12 x+17$, we take

$$
S=\left(U(x+1), V_{5} \pm 12(x+1), U(x) \pm(12 x+25), V_{9} \pm(24 x+25), U(x+1) \pm(24 x+38)\right) .
$$

In fact, $\operatorname{supp}(S)=[1,3 \rho] \backslash\{\rho, 2 \rho, 3 \rho\}$. In this case we define $\mathcal{A}$ as the sequence of the first $h$ elements of $\underset{c=0}{\stackrel{\lfloor h /|S|\rfloor}{H}}(S \pm 3 \rho c)$.

Example 4.6. Following the proof of Lemma 4.5, we obtain the following sequence $\mathcal{A}_{5,15}=$ $\left(B_{1}, \ldots, B_{5}\right)$ of shiftable blocks:

\begin{tabular}{|c|c|c|c|c|c|c|c|}
\hline \multirow{2}{*}{$B_{1}$} & & 1 & $\overline{-2}$ & 5 & -6 & \multicolumn{2}{|l|}{11} \\
\hline & & -3 & 4 & -7 & 8 & -12 & \\
\hline \multirow{2}{*}{$B_{2}$} & & 16 & 19 & -25 & 24 & \begin{tabular}{|c|}
-20 \\
\end{tabular} & -14 \\
\hline & & -18 & -17 & $\overline{23}$ & -22 & 21 & 13 \\
\hline \multirow{2}{*}{$B_{3}$} & & 26 & -27 & 31 & $\begin{array}{l}-32 \\
\end{array}$ & -35 & 3 \\
\hline & & -28 & 29 & -33 & 34 & 36 & $\begin{array}{l}-38 \\
\end{array}$ \\
\hline & & 39 & -40 & 46 & 49 & \begin{tabular}{|l|}
-43 \\
\end{tabular} & -51 \\
\hline & & -41 & 42 & -48 & -47 & 44 & 50 \\
\hline & & 52 & -53 & 56 & -57 & -61 & 63 \\
\hline & & -54 & 55 & -58 & 59 & 62 & -64 \\
\hline
\end{tabular}

Proposition 4.7. Let $m$ and $s$ be even integers with $m \geq 2, s \geq 6$ and $s \equiv 0(\bmod 6)$. Let $t$ be a divisor of $m s$ and set $\ell=\frac{2 m s}{t}+1$. There exists a sequence $\mathcal{B}$ of $\frac{m}{2}$ blocks of size $2 \times s$ such that $\mathcal{B}$ satisfies condition (4.2) and $\operatorname{supp}(\mathcal{B})=[1, m s+\lfloor t / 2\rfloor] \backslash\{j \ell: j \in[1,\lfloor t / 2\rfloor]\}$.

Proof. Write $s=6 q$, with $q \geq 1$ : by Lemma 4.5, we can construct a sequence $\mathcal{A}=\mathcal{A}_{\frac{m q}{2}, \ell}=$ $\left(A_{1}, \ldots, A_{\frac{m q}{2}}\right)$ of shiftable blocks of size $2 \times 6$ in such a way that $\mathcal{A}$ satisfies condition (4.2) and $\operatorname{supp}(\mathcal{A})=[1, m s+\lfloor t / 2\rfloor] \backslash\{j \ell: j \in[1,\lfloor t / 2\rfloor]\}$. So, for all $i \in\left[1, \frac{m}{2}\right]$, let $B_{i}$ be the block of size $2 \times s$ obtained by juxtaposing the $q$ blocks of $\mathcal{A}$

$$
A_{(i-1) q+1}, A_{(i-1) q+2}, A_{(i-1) q+3}, \ldots, A_{i q} .
$$

By construction, the sequence $\mathcal{B}=\left(B_{1}, \ldots, B_{\frac{m}{2}}\right)$ satisfies condition (4.2) and has cardinality $\frac{m}{2}$. Furthermore, since we used all the blocks of $\mathcal{A}$, we obtain that $\operatorname{supp}(\mathcal{B})=\operatorname{supp}(\mathcal{A})$.

Proposition 4.8. Let $m$ and $s$ be even integers with $m \geq 2, s \geq 8$ and $s \equiv 2(\bmod 6)$. Let $t$ be a divisor of $m s$ and set $\ell=\frac{2 m s}{t}+1$. There exists a sequence $\mathcal{B}$ of $\frac{m}{2}$ blocks of size $2 \times s$ such that $\mathcal{B}$ satisfies condition (4.2) and $\operatorname{supp}(\mathcal{B})=[1, m s+\lfloor t / 2\rfloor] \backslash\{j \ell: j \in[1,\lfloor t / 2\rfloor]\}$.

Proof. Write $s=6 q+8$ and $N=6 m q+\eta$, where $\eta=\left\lfloor\frac{6 m q}{\ell-1}\right\rfloor$. By Lemma 4.5, we can construct a sequence $\mathcal{A}=\mathcal{A}_{\frac{m q}{2}, \ell}$ satisfying condition (4.2) and whose elements are shiftable blocks of size $2 \times 6$. We also have $\operatorname{supp}(\mathcal{A})=[1, N] \backslash\{j \ell: j \in[1, \eta]\}$. Now, we have to construct a sequence $\mathcal{G}$ of shiftable blocks of size $2 \times 8$ satisfying condition (4.2) in such a way that $|\mathcal{G}|=\frac{m}{2}$ and

$$
\operatorname{supp}(\mathcal{G})=[N+1, m s+\lfloor t / 2\rfloor] \backslash\{j \ell: j \in[\eta+1,\lfloor t / 2\rfloor]\}
$$


To this purpose, we use the shiftable blocks of size $2 \times 8$ of Figure 5. Each of these blocks has rows that sum to zero and columns with the following sums:

$$
F_{3}:(-3,3,3,-3,-3,3,3,-3), \quad F_{5}, W_{i, j}, V_{i}:(-2,2,1,-1,-2,2,1,-1) .
$$

Furthermore, observe that

$$
\begin{aligned}
\operatorname{supp}\left(F_{3}\right) & =[1,24] \backslash\{3,6,9,12,15,18,21,24\}, \\
\operatorname{supp}\left(F_{5}\right) & =[1,20] \backslash\{5,10,15,20\}, \\
\operatorname{supp}\left(W_{7,3}\right) & =[1,19] \backslash\{3,10,17\}, \\
\operatorname{supp}\left(W_{7, i}\right) & =[1,18] \backslash\{i, 7+i\}, \quad i=5,7, \\
\operatorname{supp}\left(W_{9,5}\right) & =[1,18] \backslash\{5,14\}, \\
\operatorname{supp}\left(W_{11, i}\right) & =[1,18] \backslash\{i, 11+i\}, \quad i=3,5, \\
\operatorname{supp}\left(V_{j}\right) & =[1,17] \backslash\{j\}, \quad j=1,3,5,7,9,11,13,15,17 .
\end{aligned}
$$

Keeping the strategy of Lemma4.5, we describe basic sequences $S$ of blocks.

If $\ell=3$, then $t=m s$ and $N=9 m q \equiv 0(\bmod 3)$. We can take $\mathcal{G}=\stackrel{m / 2-1}{+}\left(F_{3} \pm(N+24 c)\right)$. In fact, we have $\operatorname{supp}(\mathcal{G})=\left[9 m q+1, \frac{3 m s}{2}\right] \backslash\left\{3 j: j \in\left[3 m q+1, \frac{m s}{2}\right]\right\}$, as required. If $\ell=5$, then $N=15 \frac{m q}{2} \equiv 0(\bmod 5)$ and we can take $\mathcal{G}=\underset{c=0}{\stackrel{m / 2-1}{\#}}\left(F_{5} \pm(N+20 c)\right)$. We obtain $\operatorname{supp}(\mathcal{G})=\left[15 \frac{m q}{2}+1,5 \frac{m s}{4}\right] \backslash\left\{5 j: j \in\left[\frac{3 m q}{2}+1, \frac{m s}{4}\right]\right\}$.

If $\ell=7$, then 3 divides $m$ and $N=7 m q \equiv 0(\bmod 7)$. We can take $S=\left(W_{7,7}, W_{7,3} \pm\right.$ $\left.18, W_{7,5} \pm 37\right)$, as $\operatorname{supp}(S)=[1,8 \ell] \backslash\{j \ell: j \in[1,8]\}$. Since $|S|=3$, we take $\mathcal{G}=\underset{c=0}{\stackrel{m / 6-1}{\#}}(S \pm$ $(N+8 \ell c))$.

If $\ell=13$, then 3 divides $m$ and $N=13 \frac{m q}{2} \equiv 0(\bmod 13)$. Let $S=\left(V_{13}, V_{9} \pm 17, V_{5} \pm 34\right)$ : we have $\operatorname{supp}(S)=[1,4 \ell] \backslash\{j \ell: j \in[1,4]\}$. Hence $\mathcal{G}=\underset{c=0}{\prod_{m}^{m / 6-1}}(S \pm(N+4 \ell c))$.

If $\ell=9$, we have to distinguish two cases.

(1) If $m q \equiv 0(\bmod 4)$, then $N=27 \frac{m q}{4} \equiv 0(\bmod 9)$. Hence, we can take $\mathcal{G}=\stackrel{m / 2-1}{\underset{c=0}{\#}}\left(V_{9} \pm\right.$ $(N+2 \ell c))$. Note that $\operatorname{supp}\left(V_{9}\right)=[1,2 \ell] \backslash\{\ell, 2 \ell\}$.

(2) If $m q \equiv 2(\bmod 4)$, then $N \equiv 4(\bmod 9)$. In this case, we take $\mathcal{G}=\stackrel{m}{+2}_{c=0}^{m / 2-1}\left(W_{9,5} \pm\right.$ $(N+2 \ell c))$ : recall that $\operatorname{supp}\left(W_{9,5}\right)=[1,2 \ell] \backslash\{\ell-4,2 \ell-4\}$.

For $\ell=11$ we have five possibilities.

(1) If $m q \equiv 0(\bmod 10)$ then $N \equiv 0(\bmod \ell)$, so we can take $S=\left(V_{11}, W_{11,5} \pm 17, V_{9} \pm\right.$ $\left.35, W_{11,3} \pm 52, V_{7} \pm 70\right)$. We have $\operatorname{supp}(S)=[1,8 \ell] \backslash\{j \ell: j \in[1,8]\}$.

(2) If $m q \equiv 2(\bmod 10)$, then $N \equiv 2(\bmod \ell)$, so we can take $S=\left(V_{9}, W_{11,3} \pm 17, V_{7} \pm\right.$ $\left.35, V_{11} \pm 53, W_{11,5} \pm 70\right)$. We get $\operatorname{supp}(S)=[1,8 \ell] \backslash\{j \ell-2: j \in[1,8]\}$.

(3) If $m q \equiv 4(\bmod 10)$, then $N \equiv 4(\bmod \ell)$, so we can take $S=\left(V_{7}, V_{11} \pm 18, W_{11,5} \pm\right.$ $\left.35, V_{9} \pm 53, W_{11,3} \pm 70\right)$. Note that $\operatorname{supp}(S)=[1,8 \ell] \backslash\{j \ell-4: j \in[1,8]\}$.

(4) If $m q \equiv 6(\bmod 10)$, then $N \equiv 6(\bmod \ell)$, so we can take $S=\left(W_{11,5}, V_{9} \pm 18, W_{11,3} \pm\right.$ $\left.35, V_{7} \pm 53, V_{11} \pm 71\right)$. We have $\operatorname{supp}(S)=[1,8 \ell] \backslash\{j \ell-6: j \in[1,8]\}$.

(5) If $m q \equiv 8(\bmod 10)$, then $N \equiv 8(\bmod \ell)$, so we can take $S=\left(W_{11,3}, V_{7} \pm 18, V_{11} \pm\right.$ $\left.36, W_{11,5} \pm 53, V_{9} \pm 71\right)$. We get $\operatorname{supp}(S)=[1,8 \ell] \backslash\{j \ell-8: j \in[1,8]\}$. 
In all five cases, $\mathcal{G}$ consists of the first $\frac{m}{2}$ elements of $\underset{c=0}{\stackrel{\lfloor m / 10\rfloor}{H}}(S \pm(N+8 \ell c))$.

For $\ell=15$ we have seven possibilities.

(1) If $m q \equiv 0(\bmod 14)$ then $N \equiv 0(\bmod \ell)$, so we can take $S=\left(V_{15}, V_{13} \pm 17, V_{11} \pm\right.$ $\left.34, V_{9} \pm 51, V_{7} \pm 68, V_{5} \pm 85, V_{3} \pm 102\right)$. In fact, $\operatorname{supp}(S)=[1,8 \ell] \backslash\{j \ell: j \in[1,8]\}$.

(2) If $m q \equiv 2(\bmod 14)$ then $N \equiv 12(\bmod \ell)$, so we can take $S=\left(V_{3}, V_{15} \pm 18, V_{13} \pm\right.$ $\left.35, V_{11} \pm 52, V_{9} \pm 69, V_{7} \pm 86, V_{5} \pm 103\right)$. We get $\operatorname{supp}(S)=[1,8 \ell] \backslash\{j \ell-12: j \in[1,8]\}$.

(3) If $m q \equiv 4(\bmod 14)$ then $N \equiv 10(\bmod \ell)$, so we can take $S=\left(V_{5}, V_{3} \pm 17, V_{15} \pm\right.$ $\left.35, V_{13} \pm 52, V_{11} \pm 69, V_{9} \pm 86, V_{7} \pm 103\right)$. In fact, $\operatorname{supp}(S)=[1,8 \ell] \backslash\{j \ell-10: j \in[1,8]\}$.

(4) If $m q \equiv 6(\bmod 14)$ then $N \equiv 8(\bmod \ell)$, so we can take $S=\left(V_{7}, V_{5} \pm 17, V_{3} \pm 34, V_{15} \pm\right.$ $\left.52, V_{13} \pm 69, V_{11} \pm 86, V_{9} \pm 103\right)$. We have $\operatorname{supp}(S)=[1,8 \ell] \backslash\{j \ell-8: j \in[1,8]\}$.

(5) If $m q \equiv 8(\bmod 14)$ then $N \equiv 6(\bmod \ell)$, so we can take $S=\left(V_{9}, V_{7} \pm 17, V_{5} \pm 34, V_{3} \pm\right.$ $\left.51, V_{15} \pm 69, V_{13} \pm 86, V_{11} \pm 103\right)$. In fact, $\operatorname{supp}(S)=[1,8 \ell] \backslash\{j \ell-6: j \in[1,8]\}$.

(6) If $m q \equiv 10(\bmod 14)$ then $N \equiv 4(\bmod \ell)$, so we can take $S=\left(V_{11}, V_{9} \pm 17, V_{7} \pm\right.$ $\left.34, V_{5} \pm 51, V_{3} \pm 68, V_{15} \pm 86, V_{13} \pm 103\right)$. In fact, $\operatorname{supp}(S)=[1,8 \ell] \backslash\{j \ell-4: j \in[1,8]\}$.

(7) If $m q \equiv 12(\bmod 14)$ then $N \equiv 2(\bmod \ell)$, so we can take $S=\left(V_{13}, V_{11} \pm 17, V_{9} \pm\right.$ $\left.34, V_{7} \pm 51, V_{5} \pm 68, V_{3} \pm 85, V_{15} \pm 103\right)$. In fact, $\operatorname{supp}(S)=[1,8 \ell] \backslash\{j \ell-2: j \in[1,8]\}$.

In all seven cases, $\mathcal{G}$ consists of the first $\frac{m}{2}$ elements of $\underset{c=0}{\stackrel{\lfloor m / 14\rfloor}{H}}(S \pm(N+8 \ell c))$.

Suppose now that $\ell \geq 17$ : in this case, any set of 16 consecutive integers contains at most one multiple of $\ell$. We start considering the interval $[N+1, N+16]$ and the first multiple of $\ell$ belonging to the interval $[N+1, m s+\lfloor t / 2\rfloor]$. So, if $(\eta+1) \ell$ is an element of $[N+1, N+16]$ we take the block $V_{r}$ where $r$ must be chosen in such a way that $\operatorname{supp}\left(V_{r}\right)$ does not contain $(\eta+1) \ell$. Otherwise, we take the block $V_{13}$ and repeat this process considering the interval $[N+17, N+32]$.

It will be useful to define, for all $b \geq 1$, the sequence

$$
U(b)=\left(V_{17}, V_{17} \pm 16, V_{17} \pm 32, \ldots, V_{17} \pm 16(b-1)\right) .
$$

Also, we set $U(0)$ to be the empty sequence: so, for all $b \geq 0$ the sequence $U(b)$ contains $b$ elements and $\operatorname{supp}(U(b))=[1,16 b]$.

Write $(\eta+1) \ell-N=16 h_{0}+r_{0}$, where $0 \leq r_{0}<16$, and define the sequence

$$
S_{0}=\left(U\left(h_{0}\right), V_{r_{0}} \pm 16 h_{0}\right) \text {. }
$$

Note that $r_{0}$ is odd, since $\ell$ is odd and $(\eta+1) \ell-N \equiv(\eta+1) \ell+\eta \equiv 1(\bmod 2)$. Furthermore, $\operatorname{supp}\left(S_{0} \pm N\right)=\left[N+1, N+16 h_{0}+17\right] \backslash\{(\eta+1) \ell\}$.

Now, for all $j \in[1,\lfloor t / 2\rfloor-\eta]$, write $\ell-17+r_{j-1}=16 h_{j}+r_{j}$, where $0 \leq r_{j}<16$, and define the sequence

$$
S_{j}=\left(U\left(h_{j}\right) \pm\left(17 j+16 \sum_{i=0}^{j-1} h_{i}\right), V_{r_{j}} \pm\left(17 j+16 \sum_{i=0}^{j} h_{i}\right)\right) .
$$

Note that $(\eta+j+1) \ell-N=16 \sum_{i=0}^{j} h_{i}+17 j+r_{j}$ and

$$
\operatorname{supp}\left(S_{j} \pm N\right)=\left[N+1+17 j+16 \sum_{i=0}^{j-1} h_{i}, N+17(j+1)+16 \sum_{i=0}^{j} h_{i}\right] \backslash\{(\eta+j+1) \ell\} .
$$

The elements of $\mathcal{G}$ are the first $\frac{m}{2}$ blocks in $\underset{c=0}{\stackrel{\lfloor t / 2\rfloor-\eta}{+}}\left(S_{c} \pm N\right)$. 
Finally, writing $\mathcal{A}=\left(A_{1}, \ldots, A_{\frac{m q}{2}}\right)$ and $\mathcal{G}=\left(G_{1}, \ldots, G_{\frac{m}{2}}\right)$, for all $i=1, \ldots, \frac{m}{2}$, let $B_{i}$ be the block of size $2 \times s$ obtained by juxtaposing the $q$ blocks

$$
A_{(i-1) q+1}, A_{(i-1) q+2}, A_{(i-1) q+3}, \ldots, A_{i q}
$$

and the block $G_{i}$. By construction, the sequence $\mathcal{B}=\left(B_{1}, \ldots, B_{\frac{m}{2}}\right)$ satisfies condition (4.2), has cardinality $\frac{m}{2}$ and $\operatorname{supp}(\mathcal{B})=\operatorname{supp}(\mathcal{A}) \cup \operatorname{supp}(\mathcal{G})=[1, m s+\lfloor t / 2\rfloor] \backslash\{j \ell: j \in[1,\lfloor t / 2\rfloor]\}$.

\begin{tabular}{|c|c|c|c|c|c|c|c|c|c|}
\hline \multirow{2}{*}{$F_{3}$} & \multirow{2}{*}{$=$} & 1 & -2 & -7 & 8 & 13 & -14 & -19 & 20 \\
\hline & & -4 & 5 & 10 & -11 & -16 & 17 & 22 & -23 \\
\hline \multirow{2}{*}{$F_{5}$} & \multirow{2}{*}{$=$} & 1 & -2 & 19 & -14 & 7 & -6 & 12 & -17 \\
\hline & & -3 & 4 & -18 & 13 & -9 & 8 & -11 & 16 \\
\hline \multirow{2}{*}{$W_{7,3}$} & \multirow{2}{*}{$=$} & 4 & 7 & -8 & -12 & 13 & 16 & -1 & -19 \\
\hline & & -6 & -5 & 9 & 11 & -15 & -14 & 2 & 18 \\
\hline \multirow{2}{*}{$W_{7,5}$} & \multirow{2}{*}{$=$} & 1 & -2 & -6 & 8 & -15 & -14 & 18 & 10 \\
\hline & & -3 & 4 & 7 & -9 & 13 & 16 & -17 & -11 \\
\hline \multirow{2}{*}{$W_{7,7}$} & \multirow{2}{*}{$=$} & 1 & -2 & 6 & -16 & -11 & -8 & 13 & 17 \\
\hline & & -3 & 4 & -5 & 15 & 9 & 10 & -12 & -18 \\
\hline \multirow{2}{*}{$W_{9,5}$} & \multirow{2}{*}{$=$} & 1 & -2 & -6 & 8 & -12 & 13 & -17 & 15 \\
\hline & & -3 & 4 & 7 & -9 & 10 & -11 & 18 & -16 \\
\hline \multirow{2}{*}{$W_{11,3}$} & \multirow{2}{*}{$=$} & 5 & -8 & $\overline{-1}$ & $\begin{array}{ll}-13 \\
\end{array}$ & 9 & 6 & 18 & -16 \\
\hline & & -7 & 10 & 2 & 12 & -11 & -4 & -17 & 15 \\
\hline \multirow{2}{*}{$W_{11,5}$} & \multirow{2}{*}{$=$} & 1 & -2 & $\overline{-6}$ & 8 & 13 & 14 & -10 & -18 \\
\hline & & -3 & 4 & 7 & -9 & -15 & -12 & 11 & 17 \\
\hline \multirow{2}{*}{$V_{1}$} & \multirow{2}{*}{$=$} & 2 & -3 & 17 & -13 & 7 & -6 & 11 & -15 \\
\hline & & -4 & 5 & -16 & 12 & -9 & 8 & -10 & 14 \\
\hline \multirow{2}{*}{$V_{3}$} & \multirow{2}{*}{$=$} & 4 & 7 & -1 & 14 & -12 & 13 & -8 & -17 \\
\hline & & -6 & -5 & 2 & -15 & 10 & -11 & 9 & 16 \\
\hline \multirow{2}{*}{$V_{5}$} & \multirow{2}{*}{$=$} & 1 & -2 & 17 & -13 & 7 & -6 & 11 & -15 \\
\hline & & -3 & 4 & -16 & 12 & -9 & 8 & -10 & 14 \\
\hline \multirow{2}{*}{$V_{7}$} & \multirow{2}{*}{$=$} & 1 & -8 & 6 & 16 & -11 & -2 & 13 & -15 \\
\hline & & -3 & 10 & -5 & -17 & 9 & 4 & -12 & 14 \\
\hline \multirow{2}{*}{$V_{9}$} & \multirow{2}{*}{$=$} & 1 & -2 & 17 & -13 & 6 & -5 & 11 & -15 \\
\hline & & -3 & 4 & -16 & 12 & -8 & 7 & -10 & 14 \\
\hline \multirow{2}{*}{$V_{11}$} & & 1 & -2 & 17 & -6 & -15 & -12 & 8 & 9 \\
\hline & $=$ & -3 & 4 & -16 & 5 & 13 & 14 & -7 & -10 \\
\hline$V_{13}$ & $=$ & 1 & -2 & 17 & 9 & -7 & 8 & -11 & -15 \\
\hline & & -3 & 4 & -16 & -10 & 5 & -6 & 12 & 14 \\
\hline$V_{15}$ & $=$ & 1 & -2 & -5 & 7 & 11 & 14 & -16 & -10 \\
\hline 15 & - & -3 & 4 & 6 & -8 & -13 & -12 & 17 & 9 \\
\hline$V_{17}$ & $=$ & 1 & -2 & 16 & -12 & 6 & -5 & 10 & -14 \\
\hline & - & -3 & 4 & -15 & 11 & -8 & 7 & -9 & 13 \\
\hline
\end{tabular}

FiguRE 5. Shiftable blocks of size $2 \times 8$ satisfying condition (4.2). 
Proposition 4.9. Let $m$ and $s$ be even integers with $m \geq 2, s \geq 10$ and $s \equiv 4(\bmod 6)$. Let $t$ be a divisor of $m s$ and set $\ell=\frac{2 m s}{t}+1$. There exists a sequence $\mathcal{B}$ of $\frac{m}{2}$ blocks of size $2 \times s$ such that $\mathcal{B}$ satisfies condition (4.2) and $\operatorname{supp}(\mathcal{B})=[1, m s+\lfloor t / 2\rfloor] \backslash\{j \ell: j \in[1,\lfloor t / 2\rfloor]\}$.

Proof. This proof is very similar to that of Proposition 4.8, so we can skip some details. Write $s=6 q+10$ and $N=6 m q+\eta$, where $\eta=\left\lfloor\frac{6 m q}{\ell-1}\right\rfloor$. Let $\mathcal{A}=\mathcal{A}_{\frac{m q}{2}, \ell}$ be the sequence satisfying condition (4.2) constructed in Lemma 4.5. We have to construct a sequence $\mathcal{G}$ of shiftable blocks of size $2 \times 10$ satisfying condition (4.2) in such a way that $|\mathcal{G}|=\frac{m}{2}$ and

$$
\operatorname{supp}(\mathcal{G})=[N+1, m s+\lfloor t / 2\rfloor] \backslash\{j \ell: j \in[\eta+1,\lfloor t / 2\rfloor]\} .
$$

To this purpose, we use the shiftable blocks of size $2 \times 10$ of Figure 6 . Each of these blocks has rows that sum to zero and columns with the following sums:

$$
\begin{gathered}
F_{3}:(-1,1,-3,3,6,-6,-3,3,3,-3), \quad F_{5}:(-2,2,-2,2,1,-1,-2,2,2,-2), \\
W_{7, i}:(1,-1,-2,2,1,-1,-2,2,2,-2), \\
W_{9, i}, W_{13,5}, W_{15, i}, V_{j}:(-2,2,-2,2,1,-1,-2,2,2,-2) .
\end{gathered}
$$

Observe that

$$
\begin{aligned}
\operatorname{supp}\left(F_{3}\right) & =[1,30] \backslash\{3,6,9,12,15,18,21,24,27,30\}, \\
\operatorname{supp}\left(F_{5}\right) & =[1,25] \backslash\{5,10,15,20,25\}, \\
\operatorname{supp}\left(W_{7, i}\right) & =[1,23] \backslash\{i, 7+i, 14+i\}, \quad i=3,5,7, \\
\operatorname{supp}\left(W_{9, i}\right) & =[1,22] \backslash\{i, 9+i\}, \quad i=5,9, \\
\operatorname{supp}\left(W_{13,5}\right) & =[1,22] \backslash\{5,18\}, \\
\operatorname{supp}\left(W_{15, i}\right) & =[1,22] \backslash\{i, 15+i\}, \quad i=3,5, \\
\operatorname{supp}\left(V_{j}\right) & =[1,21] \backslash\{j\}, \quad j=1,3,5,7,9,11,13,15,17,19,21 .
\end{aligned}
$$

Furthermore, if $\ell \in\{3,5,7,11,13,19\}$, then $N \equiv 0(\bmod \ell)$.

If $\ell=3$, let $S=\left(F_{3}\right)$ and $\mathcal{G}=\underset{c=0}{\# / 2-1}(S \pm(N+24 c))$. Similarly, if $\ell=5,11$, let $S=\left(F_{5}\right)$ and $S=\left(V_{11}\right)$ respectively.

If $\ell=7$, let $S=\left(W_{7,7}, W_{7,5} \pm 23, W_{7,3} \pm 46\right)$. Note that $\operatorname{supp}(S)=[1,10 \ell] \backslash\{j \ell: j \in[1,10]\}$.

In this case, $\mathcal{G}=\underset{c=0}{\stackrel{m}{+}+6-1}(S \pm(N+10 \ell c))$.

If $\ell=13$, let $S=\left(V_{13}, W_{13,5} \pm 21, V_{9} \pm 43\right)$. Note that $\operatorname{supp}(S)=[1,5 \ell] \backslash\{j \ell: j \in[1,5]\}$. We can take $\mathcal{G}=\underset{c=0}{m / 6-1}(S \pm(N+5 \ell c))$.

If $\ell=19$ let

$$
S=\left(V_{19}, V_{17} \pm 21, V_{15} \pm 42, V_{13} \pm 63, V_{11} \pm 84, V_{9} \pm 105, V_{7} \pm 126, V_{5} \pm 147, V_{3} \pm 168\right) .
$$

We have $\operatorname{supp}(S)=[1,10 \ell] \backslash\{j \ell: j \in[1,10]\}$ and $\mathcal{G}={\underset{c=0}{m / 18-1}}_{+}^{m}(S \pm(N+10 \ell c))$.

For $\ell=9$ we have two possibilities.

(1) If $m q \equiv 0(\bmod 4)$, then $N \equiv 0(\bmod \ell)$ and we can take $S=\left(W_{9,9}, W_{9,5} \pm 22\right)$. In fact, $\operatorname{supp}(S)=[1,5 \ell] \backslash\{j \ell: j \in[1,5]\}$.

(2) If $m q \equiv 2(\bmod 4)$, then $N \equiv 4(\bmod \ell)$. We can take $S=\left(W_{9,5}, W_{9,9} \pm 23\right)$, in fact $\operatorname{supp}(S)=[1,5 \ell] \backslash\{j \ell-4: j \in[1,5]\}$.

In both cases, the sequence $\mathcal{G}$ consists of the first $\frac{m}{2}$ elements of $\underset{c=0}{\stackrel{\lfloor m / 4\rfloor}{\#}}(S \pm(N+5 \ell c))$.

If $\ell=15$ we have seven possibilities. 
(1) If $m q \equiv 0(\bmod 14)$ then $N \equiv 0(\bmod \ell)$ and we can take $S=\left(V_{15}, V_{9} \pm 21, W_{15,3} \pm\right.$ $\left.42, V_{11} \pm 64, W_{15,5} \pm 85, V_{13} \pm 107, V_{7} \pm 128\right)$. In fact, $\operatorname{supp}(S)=[1,10 \ell] \backslash\{j \ell: j \in[1,10]\}$.

(2) If $m q \equiv 2(\bmod 14)$ then $N \equiv 12(\bmod \ell)$ and we can take $S=\left(W_{15,3}, V_{11} \pm\right.$ $\left.22, W_{15,5} \pm 43, V_{13} \pm 65, V_{7} \pm 86, V_{15} \pm 108, V_{9} \pm 129\right)$. In fact, $\operatorname{supp}(S)=[1,10 \ell] \backslash\{j \ell-12:$ $j \in[1,10]\}$.

(3) If $m q \equiv 4(\bmod 14)$ then $N \equiv 10(\bmod \ell)$ and we can take $S=\left(W_{15,5}, V_{13} \pm 22, V_{7} \pm\right.$ $\left.43, V_{15} \pm 65, V_{9} \pm 86, W_{15,3} \pm 107, V_{11} \pm 129\right)$. In fact, $\operatorname{supp}(S)=[1,10 \ell] \backslash\{j \ell-10: j \in$ $[1,10]\}$.

(4) If $m q \equiv 6(\bmod 14)$ then $N \equiv 8(\bmod \ell)$ and we can take $S=\left(V_{7}, V_{15} \pm 22, V_{9} \pm\right.$ $\left.43, W_{15,3} \pm 64, V_{11} \pm 86, W_{15,5} \pm 107, V_{13} \pm 129\right)$. In fact, $\operatorname{supp}(S)=[1,10 \ell] \backslash\{j \ell-8:$ $j \in[1,10]\}$.

(5) If $m q \equiv 8(\bmod 14)$ then $N \equiv 6(\bmod \ell)$ and we can take $S=\left(V_{9}, W_{15,3} \pm 21, V_{11} \pm\right.$ $\left.43, W_{15,5} \pm 64, V_{13} \pm 86, V_{7} \pm 107, V_{15} \pm 129\right)$. In fact, $\operatorname{supp}(S)=[1,10 \ell] \backslash\{j \ell-6: j \in$ $[1,10]\}$.

(6) If $m q \equiv 10(\bmod 14)$ then $N \equiv 4(\bmod \ell)$ and we can take $S=\left(V_{11}, W_{15,5} \pm 21, V_{13} \pm\right.$ $\left.43, V_{7} \pm 64, V_{15} \pm 86, V_{9} \pm 107, W_{15,3} \pm 128\right)$. In fact, $\operatorname{supp}(S)=[1,10 \ell] \backslash\{j \ell-4: j \in$ $[1,10]\}$.

(7) If $m q \equiv 12(\bmod 14)$ then $N \equiv 2(\bmod \ell)$ and we can take $S=\left(V_{13}, V_{7} \pm 21, V_{15} \pm\right.$ $\left.43, V_{9} \pm 64, W_{15,3} \pm 85, V_{11} \pm 107, W_{15,5} \pm 128\right)$. In fact, $\operatorname{supp}(S)=[1,10 \ell] \backslash\{j \ell-2:$ $j \in[1,10]\}$.

In all seven cases, the sequence $\mathcal{G}$ consists of the first $\frac{m}{2}$ elements of $\overbrace{c=0}^{\lfloor m / 14\rfloor}(S \pm(N+10 \ell c))$. If $\ell=17$ we have four possibilities.

(1) If $m q \equiv 0(\bmod 8)$ then $N \equiv 0(\bmod \ell)$ and we can take $S=\left(V_{17}, V_{13} \pm 21, V_{9} \pm\right.$ $\left.42, V_{5} \pm 63\right)$. We have $\operatorname{supp}(S)=[1,5 \ell] \backslash\{j \ell: j \in[1,5]\}$.

(2) If $m q \equiv 2(\bmod 8)$ then $N \equiv 12(\bmod \ell)$ and we can take $S=\left(V_{5}, V_{17} \pm 22, V_{13} \pm\right.$ $\left.43, V_{9} \pm 64\right)$. We get $\operatorname{supp}(S)=[1,5 \ell] \backslash\{j \ell-12: j \in[1,5]\}$.

(3) If $m q \equiv 4(\bmod 8)$ then $N \equiv 8(\bmod \ell)$ and we can take $S=\left(V_{9}, V_{5} \pm 21, V_{17} \pm\right.$ $\left.43, V_{13} \pm 64\right)$. We have $\operatorname{supp}(S)=[1,5 \ell] \backslash\{j \ell-8: j \in[1,5]\}$.

(4) If $m q \equiv 6(\bmod 8)$ then $N \equiv 4(\bmod \ell)$ and we can take $S=\left(V_{13}, V_{9} \pm 21, V_{5} \pm\right.$ $\left.42, V_{17} \pm 64\right)$. We obtain $\operatorname{supp}(S)=[1,5 \ell] \backslash\{j \ell-4: j \in[1,5]\}$.

In all four cases, the sequence $\mathcal{G}$ consists of the first $\frac{m}{2}$ elements of $\underset{c=0}{\stackrel{\lfloor m / 8\rfloor}{\#}}(S \pm(N+5 \ell c))$.

Suppose now that $\ell \geq 21$ : in this case, any set of 20 consecutive integers contains at most one multiple of $\ell$. For all $b \geq 1$ we define the sequence

$$
U(b)=\left(V_{21}, V_{21} \pm 20, V_{21} \pm 40, \ldots, V_{21} \pm 20(b-1)\right) .
$$

Also, we set $U(0)$ to be the empty sequence: so, for all $b \geq 0$ the sequence $U(b)$ contains $b$ elements and $\operatorname{supp}(U(b))=[1,20 b]$.

Write $(\eta+1) \ell-N=20 h_{0}+r_{0}$, where $0 \leq r_{0}<20$, and define the sequence

$$
S_{0}=\left(U\left(h_{0}\right), V_{r_{0}} \pm 20 h_{0}\right) .
$$

Hence, $\operatorname{supp}\left(S_{0} \pm N\right)=\left[N+1, N+20 h_{0}+21\right] \backslash\{(\eta+1) \ell\}$. For all $j \in[1,\lfloor t / 2\rfloor-\eta]$, write $\ell-21+r_{j-1}=20 h_{j}+r_{j}$, where $0 \leq r_{j}<20$, and define 


$$
S_{j}=\left(U\left(h_{j}\right) \pm\left(21 j+20 \sum_{i=0}^{j-1} h_{i}\right), V_{r_{j}} \pm\left(21 j+20 \sum_{i=0}^{j} h_{i}\right)\right) .
$$

Note that $(\eta+j+1) \ell-N=20 \sum_{i=0}^{j} h_{i}+21 j+r_{j}$ and

$$
\operatorname{supp}\left(S_{j} \pm N\right)=\left[N+1+21 j+20 \sum_{i=0}^{j-1} h_{i}, N+21(j+1)+20 \sum_{i=0}^{j} h_{i}\right] \backslash\{(\eta+j+1) \ell\} .
$$

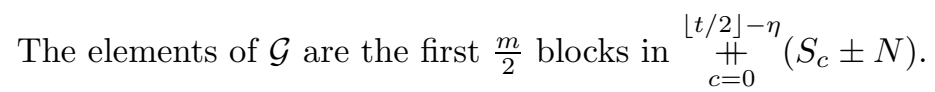

Finally, writing $\mathcal{A}=\left(A_{1}, \ldots, A_{\frac{m q}{2}}\right)$ and $\mathcal{G}=\left(G_{1}, \ldots, G_{\frac{m}{2}}\right)$, for all $i=1, \ldots, \frac{m}{2}$, let $B_{i}$ be the block of size $2 \times s$ obtained by juxtaposing the $q$ blocks

$$
A_{(i-1) q+1}, A_{(i-1) q+2}, A_{(i-1) q+3}, \ldots, A_{i q}
$$

and the block $G_{i}$. By construction, the sequence $\mathcal{B}=\left(B_{1}, \ldots, B \frac{m}{2}\right)$ satisfies condition (4.2), has cardinality $\frac{m}{2}$ and $\operatorname{supp}(\mathcal{B})=\operatorname{supp}(\mathcal{A}) \cup \operatorname{supp}(\mathcal{G})=[1, m s+\lfloor t / 2\rfloor] \backslash\{j \ell: j \in[1,\lfloor t / 2\rfloor]\}$.

Corollary 4.10. Let $m$ and $s$ be even integers with $m \geq 2, s \geq 6$ and $s \equiv 2(\bmod 4)$. Let $t$ be a divisor of $2 m s$ and set $\ell=\frac{2 m s}{t}+1$. There exists a sequence $\mathcal{B}$ of cardinality $\frac{m}{2}$ such that $\mathcal{B}$ consists of blocks of size $2 \times s$, satisfies condition (4.2) and $\operatorname{supp}(\mathcal{B})=[1, m s+\lfloor t / 2\rfloor] \backslash$ $\{j \ell: j \in[1,\lfloor t / 2\rfloor]\}$.

Proof. By the assumptions on $s$, we can write $s=2 p h$, for a suitable odd prime $p$ and a positive integer $h$. If $t$ divides $m s$, the result follows from Propositions 4.7, 4.8 and 4.9. depending on the residue class of $s$ modulo 6 . It $t$ does not divide $m s$, then $t \equiv 0(\bmod 8)$. If $t$ divides $4 m h=\frac{2 m s}{p}$, then the statement follows from Lemma 4.3, If $t$ does not divide $4 m h$, then $t$ is divisible by $p$ and so we can apply Lemma 4.1 .

Now, we arrange the blocks so far constructed, proving the following.

Proposition 4.11. Suppose $4 \leq s \leq n, 4 \leq k \leq m$ and $m s=n k$. Let $t$ be a divisor of $2 m s$.

(1) If $s \equiv 2(\bmod 4)$ and $k \equiv 0(\bmod 4)$, then there exists an integer $\mathrm{H}_{t}(m, n ; s, k)$ if and only if $m$ is even.

(2) If $s \equiv 0(\bmod 4)$ and $k \equiv 2(\bmod 4)$, then there exists an integer $\mathrm{H}_{t}(m, n ; s, k)$ if and only if $n$ is even.

Proof. (1) By Proposition 1.2 we only have to prove the existence of an integer $\mathrm{H}_{t}(m, n ; s, k)$ when $m$ is even. So, let $\mathcal{B}$ be the sequence obtained in Corollary 4.10. Set $d=\operatorname{gcd}\left(\frac{m}{2}, n\right)$ and $a=\frac{s d}{n}$. Note that $a$ is even integer. In fact, write $m=2 \bar{m} d$ and $n=d \bar{n}$. Since $k \equiv 0$ $(\bmod 4)$, from $\frac{s}{2} \cdot \frac{m}{2}=n \frac{k}{4}$ we obtain that $\bar{n}$ divides $\frac{s}{2}$.

Given a block $B_{h} \in \mathcal{B}$, define for every $j \in[1, \bar{n}]$ the block $T_{j}\left(B_{h}\right)$ of size $2 \times a$ consisting of the columns $C_{i}$ of $B_{h}$ with $i \in[a(j-1)+1, a j]$. So, the block $B_{h}$ of size $2 \times s$ is obtained juxtaposing the blocks $T_{1}\left(B_{h}\right), T_{2}\left(B_{h}\right), \ldots, T_{\bar{n}}\left(B_{h}\right)$. Furthermore, for all $i \in[1, \bar{m}]$ and all $j \in[1, \bar{n}]$, each of the sequences

$$
\left(T_{j}\left(B_{(i-1) d+1}\right), T_{j}\left(B_{(i-1) d+2}\right), \ldots, T_{j}\left(B_{i d}\right)\right),
$$

of cardinality $d$, satisfies condition (4.1). 


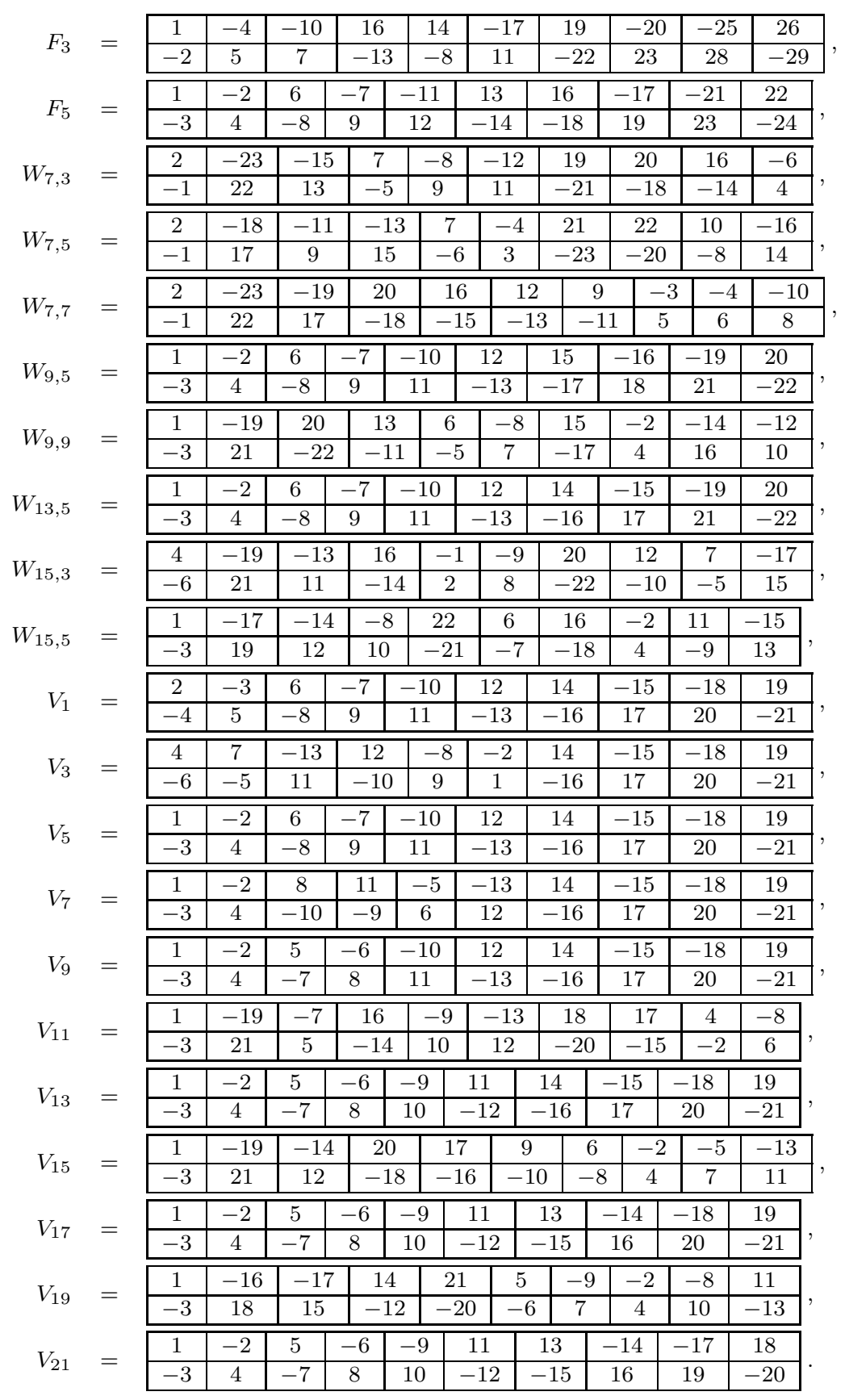

FiguRE 6 . Shiftable blocks of size $2 \times 10$ satisfying condition (4.2). 
Let $A$ be an empty array of size $\bar{m} \times \bar{n}$. For every $i \in[1, \bar{m}]$ and $j \in[1, \bar{n}]$, replace the cell $(i, j)$ of $A$ with the block $P\left(T_{j}\left(B_{(i-1) d+1}\right), T_{j}\left(B_{(i-1) d+2}\right), \ldots, T_{j}\left(B_{i d}\right)\right)$ according to the definition of page 7. Note that, for all $r \in\left[1, \frac{m}{2}\right]$, we have $\tau_{r}(A)=\tau_{1}\left(B_{r}\right)=0$ and $\tau_{r+\frac{m}{2}}(A)=\tau_{2}\left(B_{r}\right)=0$.

By construction, $A$ is a p.f. array of size $m \times n$, its support coincides with $\operatorname{supp}(\mathcal{B})$ and its rows and columns sum to zero. Furthermore, each row contains $a \bar{n}=s$ elements and each column contains $2 a \bar{m}=k$ elements. We conclude that $A$ is an integer $\mathrm{H}_{t}(m, n ; s, k)$.

(2) It follows from (1). In fact, if $s \equiv 0(\bmod 4)$ and $k \equiv 2(\bmod 4)$, an integer $\mathrm{H}_{t}(m, n ; s, k)$ can be obtained simply by taking the transpose of an integer $\mathrm{H}_{t}(n, m ; k, s)$.

We exhibit an integer $\mathrm{H}_{12}(20,15 ; 6,8)$ and an integer $\mathrm{H}_{5}(6,15 ; 10,4)$ in Figure 7 . To help the reader, we highlighted the subarray $P\left(T_{1}\left(B_{1}\right), \ldots, T_{1}\left(B_{5}\right)\right)$ in the first case, and the subarray $P\left(T_{1}\left(B_{1}\right), T_{1}\left(B_{2}\right), T_{1}\left(B_{3}\right)\right)$ in the second case.

\section{THE CASE $s, k \equiv 2(\bmod 4)$}

The last case we consider is when $s, k \equiv 2(\bmod 4)$. We also assume that $m$ is even: this means that also $n$ must be even. We start constructing sequences $\mathcal{B}$ satisfying the following property:

fixed $2 b$ integers $\sigma_{1}, \ldots, \sigma_{2 b}$ such that $\sum_{i=1}^{b} \sigma_{2 i-1}=\sum_{i=1}^{b} \sigma_{2 i}=0$, the elements of $\mathcal{B}$ are shiftable blocks $B$ of size $2 \times 2 b$ such that $\tau_{1}(B)=\tau_{2}(B)=0$ and $\gamma_{i}(B)=\sigma_{i}$ for all $i \in[1,2 b]$.

Lemma 5.1. Let $m$ and $s$ be even integers with $m \geq 2, s \geq 6$ and $s \equiv 2(\bmod 4)$. Let $t$ be $a$ divisor of $2 m s$ and set $\ell=\frac{2 m s}{t}+1$. There exists a sequence $\mathcal{B}^{\prime}$ of $\frac{m}{2}$ shiftable blocks of size $2 \times s$ such that $\mathcal{B}^{\prime}$ satisfies condition (5.1) and $\operatorname{supp}\left(\mathcal{B}^{\prime}\right)=[1, m s+\lfloor t / 2\rfloor] \backslash\{j \ell: j \in[1,\lfloor t / 2\rfloor]\}$.

Proof. Suppose first that $t$ divides $m s$. If $s \equiv 0(\bmod 6)$, consider the shiftable blocks of size $2 \times 6$ given in Figure 8 . We can repeat the same proofs of Lemma 4.5 and Proposition 4.7, using the blocks of Figure 8 instead of those of Figure 4. In fact, each of these new blocks satisfies (5.1) and has the following column sums:

$$
F_{i}:(-1,3,-6,3,7,-6), \quad V_{j}:(-1,4,-1,-2,2,-2) .
$$

Furthermore, the new blocks have the same support of the old ones.

The same can be done for $s \equiv 2(\bmod 6)$, using the blocks of Figure 9 and the proof of Proposition 4.8. Each of these new blocks satisfies (5.1) and has the following column sums: $(-1,-8,-12,4,10,-1,3,5)$. Finally, for $s \equiv 4(\bmod 6)$ we can repeat the proof of Proposition 4.9 using the blocks of Figure 10. Each of these new blocks satisfies (5.1) and has the following column sums:

$$
F_{i}=(-1,-1,1,7,6,3,-3,-2,-3,-7), \quad W_{j, i}, V_{j}=(-1,4,-1,-2,2,-2,-2,2,2,-2) .
$$

Suppose now that $t$ does not divide $m s$. In this case, repeat the proofs of Lemmas 4.1 and 4.3. using the blocks of Figures 11 and 12 instead of those of Figures 2 and 3 respectively. Note that the blocks of Figure 11 satisfy property (5.1) with column sums $(-2 \ell, 2 \ell, 2 \ell,-2 \ell)$ and $(-\ell, 4 \ell,-\ell,-2 \ell, 2 \ell,-2 \ell)$. The blocks $W_{4}, W_{6}$ of Figure 12 satisfy property (5.1) with column sums $(-y, y, y,-y)$ and $(-y,(p-2) y+1,2 y,-(2 p-1) y-2,-y,(p+1) y+1)$, respectively.

In all these cases, we obtain a sequence $\mathcal{B}^{\prime}$ that satisfies (5.1) and has the required support. 

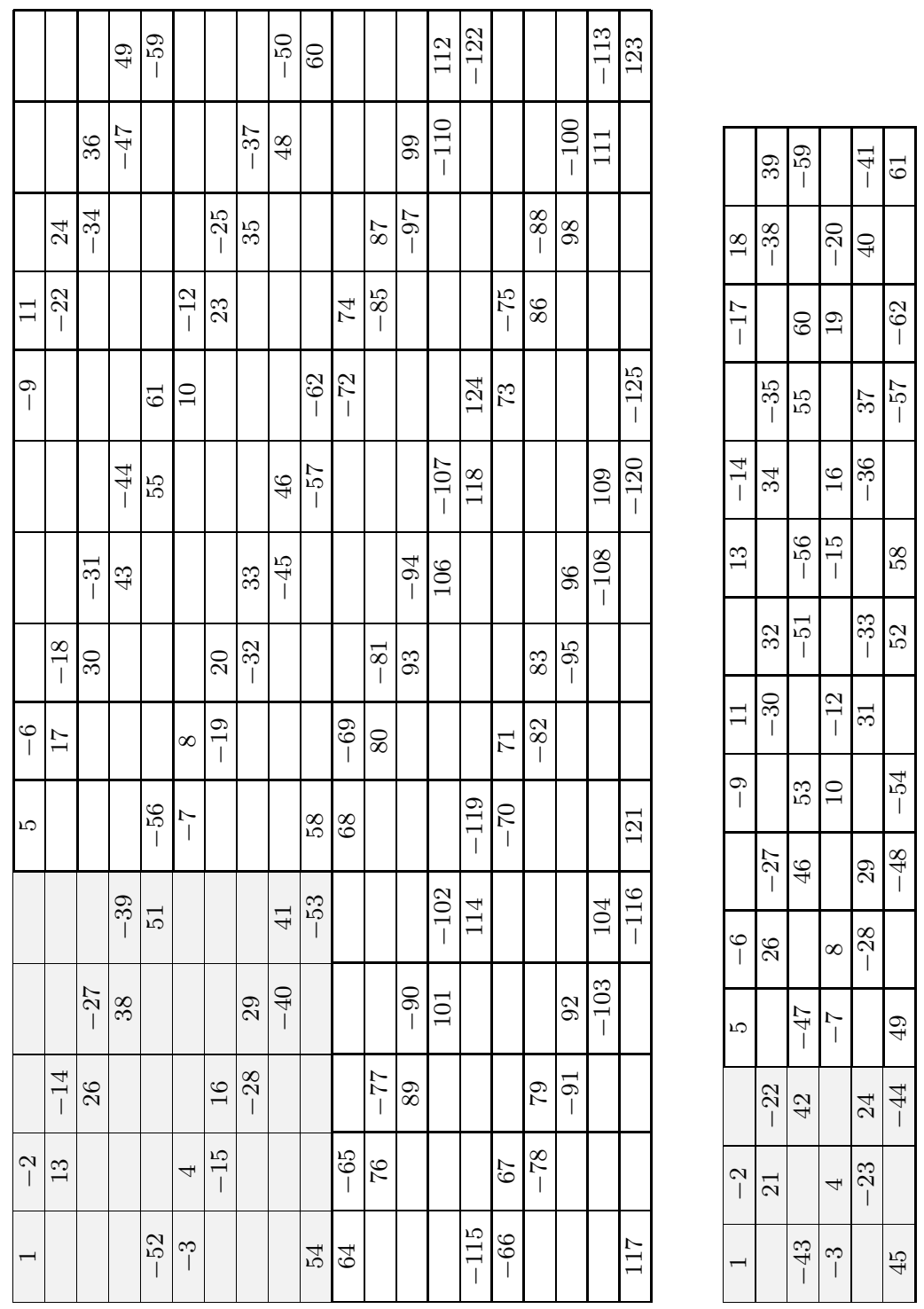

Figure 7. An integer $\mathrm{H}_{12}(20,15 ; 6,8)$ (on the left) and an integer $\mathrm{H}_{5}(6,15$; $10,4)$ (on the right).

Proposition 5.2. Suppose $6 \leq s \leq n, 6 \leq k \leq m, m s=n k$ and $s, k \equiv 2(\bmod 4)$. If $m$ is even, there exists a shiftable integer $\mathrm{H}_{t}(m, n ; s, k)$ for every divisor $t$ of $2 m s$.

Proof. Without loss of generality, we may assume $m \geq n$ (and so $s \leq k)$. Let $\mathcal{B}=\left(B_{1}, \ldots, B_{\frac{m}{2}}\right)$ and $\mathcal{B}^{\prime}=\left(B_{1}^{\prime}, \ldots, B_{\frac{m}{2}}^{\prime}\right)$ be the sequences of blocks of size $2 \times s$ constructed in Corollary 4.10 and Lemma 5.1 respectively, where $\mathcal{B}$ satisfies (4.2), $\mathcal{B}^{\prime}$ satisfies (5.1) and $\operatorname{supp}(\mathcal{B})=\operatorname{supp}\left(\mathcal{B}^{\prime}\right)=$ 


\begin{tabular}{|c|c|c|c|c|c|c|c|c|c|c|c|c|c|c|c|}
\hline \multirow{2}{*}{$F_{3}$} & & 1 & -4 & -11 & 16 & -10 & 8 & \multirow[b]{2}{*}{$F_{5}$} & \multirow[b]{2}{*}{$=$} & 1 & -3 & $\overline{7}$ & -9 & -4 & 8 \\
\hline & & \begin{tabular}{l|}
-2 \\
\end{tabular} & 7 & 5 & -13 & 17 & -14 & & & -2 & 6 & -13 & 12 & 11 & -14 \\
\hline \multirow{2}{*}{$V_{1}$} & & 2 & -4 & -6 & -12 & 9 & 11 & \multirow{2}{*}{$V_{3}$} & \multirow{2}{*}{$=$} & 1 & \begin{tabular}{|l|}
-9 \\
\end{tabular} & 11 & -7 & -4 & 8 \\
\hline & & \begin{tabular}{l|}
-3 \\
\end{tabular} & 8 & 5 & 10 & -7 & -13 & & & -2 & 13 & -12 & 5 & 6 & -10 \\
\hline \multirow{2}{*}{$V_{5}$} & & -13 & 11 & 9 & -8 & \begin{tabular}{l|l}
-1 \\
\end{tabular} & 2 & \multirow{2}{*}{$V_{7}$} & \multirow{2}{*}{$=$} & 1 & 12 & 9 & -5 & -4 & -13 \\
\hline & & 12 & -7 & -10 & 6 & 3 & -4 & & & -2 & -8 & -10 & \begin{tabular}{|l|}
3 \\
\end{tabular} & $\begin{array}{l}6 \\
\end{array}$ & 11 \\
\hline \multirow{2}{*}{$V_{9}$} & & 1 & \begin{tabular}{|l|}
-3 \\
\end{tabular} & -5 & 6 & 13 & -12 & \multirow{2}{*}{$V_{11}$} & \multirow[b]{2}{*}{$=$} & 1 & -9 & 7 & -6 & \begin{tabular}{|l|}
-3 \\
\end{tabular} & 10 \\
\hline & $=$ & \begin{tabular}{l|}
-2 \\
\end{tabular} & 7 & 4 & -8 & -11 & 10 & & & -2 & 13 & \begin{tabular}{|l|}
-8 \\
\end{tabular} & 4 & 5 & \begin{tabular}{ll|}
-12 \\
\end{tabular} \\
\hline \multirow{2}{*}{$V_{13}$} & & 1 & \begin{tabular}{|l|}
-3 \\
\end{tabular} & -5 & 6 & 12 & -11 & & & & & & & & \\
\hline & & \begin{tabular}{l|}
-2 \\
\end{tabular} & 7 & 4 & -8 & -10 & 9 & & & & & & & & \\
\hline
\end{tabular}

FIGURE 8. Shiftable blocks of size $2 \times 6$ satisfying condition (5.1).

$[1, m s+\lfloor t / 2\rfloor] \backslash\left\{j \ell: j \in[1,\lfloor t / 2\rfloor]\right.$ with $\ell=\frac{2 m s}{t}+1$. Set

$$
\widetilde{\mathcal{B}}=\left(B_{\frac{n}{2}+1}, \ldots, B_{\frac{m}{2}}\right) \quad \text { and } \quad \widetilde{\mathcal{B}}^{\prime}=\left(B_{1}^{\prime}, \ldots, B_{\frac{n}{2}}^{\prime}\right) .
$$

Since $\operatorname{supp}\left(B_{i}\right)=\operatorname{supp}\left(B_{i}^{\prime}\right)$ for all $i \in\left[1, \frac{m}{2}\right]$, it follows that

$$
\operatorname{supp}\left(\widetilde{\mathcal{B}}^{\prime}\right)=\left[1, s n+\left\lfloor\frac{s n}{\ell-1}\right\rfloor\right] \backslash\left\{j \ell: j \in\left[1,\left\lfloor\frac{s n}{\ell-1}\right\rfloor\right]\right\}
$$

and

$$
\begin{aligned}
\operatorname{supp}(\widetilde{\mathcal{B}}) & =\operatorname{supp}(\mathcal{B}) \backslash \operatorname{supp}\left(\widetilde{\mathcal{B}}^{\prime}\right) \\
& =\left[s n+\left\lfloor\frac{s n}{\ell-1}\right\rfloor+1, m s+\left\lfloor\frac{t}{2}\right\rfloor\right] \backslash\left\{j \ell: j \in\left[\left\lfloor\frac{s n}{\ell-1}\right\rfloor+1,\left\lfloor\frac{t}{2}\right\rfloor\right]\right\} .
\end{aligned}
$$

Hence, $\operatorname{supp}\left(\widetilde{\mathcal{B}^{\prime}}+\widetilde{\mathcal{B}}\right)=[1, m s+\lfloor t / 2\rfloor] \backslash\{j \ell: j \in[1,\lfloor t / 2\rfloor]$.

Using the blocks of the sequence $\widetilde{\mathcal{B}}^{\prime}$, we first construct a square shiftable p.f. array $A_{1}$ of size $n$ such that each row and each column contains $s$ filled cells and such that the elements in every row and column sum to zero. Hence, take an empty array $A_{1}$ of size $n \times n$ and arrange the $\frac{n}{2}$ blocks $B_{r}^{\prime}=\left(b_{i, j}^{(r)}\right)$ of $\widetilde{\mathcal{B}^{\prime}}$ in such a way that the element $b_{1,1}^{(r)}$ fills the cell $(2 r-1,2 r-1)$ of $A_{1}$. This process makes $A_{1}$ a p.f. array with $s$ filled cells in each row and in each column. Since the rows of the blocks $B_{r}^{\prime}$ sum to zero, also the rows of $A_{1}$ sum to zero. Looking at the columns, the $s$ elements of a column of $A_{1}$ are

$$
b_{1, s}^{(r)}, b_{2, s}^{(r)}, b_{1, s-2}^{(r+1)}, b_{2, s-2}^{(r+1)}, b_{1, s-4}^{(r+2)}, b_{2, s-4}^{(r+2)}, \ldots, b_{1,2}^{(r+s / 2)}, b_{2,2}^{(r+s / 2)}
$$

or

$$
b_{1, s-1}^{(r)}, b_{2, s-1}^{(r)}, b_{1, s-3}^{(r+1)}, b_{2, s-3}^{(r+1)}, b_{1, s-5}^{(r+2)}, b_{2, s-5}^{(r+2)}, \ldots, b_{1,1}^{(r+s / 2)}, b_{2,1}^{(r+s / 2)},
$$

where the exponents $r, \ldots, r+s / 2$ must be read modulo $\frac{n}{2}$. Since $\widetilde{\mathcal{B}^{\prime}}$ satisfies condition (5.1), the sum of these elements is

$$
\sum_{j=1}^{s / 2} \sigma_{2 j}=0 \quad \text { or } \quad \sum_{j=1}^{s / 2} \sigma_{2 j-1}=0, \quad \text { respectively. }
$$

By construction, $\operatorname{supp}\left(A_{1}\right)=\operatorname{supp}\left(\widetilde{\mathcal{B}^{\prime}}\right)$.

Now, if $m=n$, then $A_{1}$ is actually a shiftable integer $\mathrm{H}_{t}(m, n ; k, s)$. Suppose that $m>n$. If we arrange the blocks of the sequence $\widetilde{\mathcal{B}}$ mimicking what we did for the construction of an 


\begin{tabular}{|c|c|c|c|c|c|c|c|c|c|}
\hline \multirow{2}{*}{$F_{3}$} & \multirow{2}{*}{$=$} & 1 & 11 & -17 & -16 & 14 & 22 & -7 & -8 \\
\hline & & -2 & -19 & 5 & 20 & -4 & -23 & 10 & 13 \\
\hline \multirow{2}{*}{$F_{5}$} & \multirow{2}{*}{$=$} & 1 & 3 & $\frac{-19}{-19}$ & 16 & -4 & 17 & -6 & -8 \\
\hline & & -2 & -11 & 7 & -12 & 14 & -18 & $\begin{array}{l}9 \\
\end{array}$ & 13 \\
\hline \multirow{2}{*}{$W_{7,3}$} & \multirow{2}{*}{$=$} & 1 & 8 & -18 & $\begin{array}{l}-9 \\
\end{array}$ & 15 & -12 & -4 & $\overline{19}$ \\
\hline & & -2 & -16 & 6 & 13 & -5 & 11 & 7 & -14 \\
\hline \multirow{2}{*}{$W_{7,5}$} & \multirow[b]{2}{*}{$=$} & $\overline{1}$ & $\overline{9}$ & $\overline{-16}$ & 18 & 13 & $\overline{-7}$ & $2-8$ & $\overline{c-10}$ \\
\hline & & -2 & -17 & 4 & -14 & -3 & 6 & 11 & 15 \\
\hline \multirow{2}{*}{$W_{7,7}$} & \multirow{2}{*}{$=$} & 1 & 3 & -17 & -4 & -6 & -10 & 15 & 18 \\
\hline & & -2 & -11 & 5 & 8 & 16 & 9 & -12 & -13 \\
\hline \multirow{2}{*}{$W_{9,5}$} & \multirow{2}{*}{$=$} & $\overline{11}$ & -17 & $\overline{-15}$ & 12 & $\overline{16}$ & -11 & -4 & 18 \\
\hline & & -2 & 9 & 3 & -8 & -6 & 10 & 7 & -13 \\
\hline \multirow{2}{*}{$W_{11,3}$} & \multirow{2}{*}{$=$} & 1 & -12 & -17 & 15 & \begin{tabular}{|c|}
-6 \\
\end{tabular} & -9 & 10 & 18 \\
\hline & & -2 & 4 & 5 & -11 & 16 & 8 & -7 & -13 \\
\hline \multirow{2}{*}{$W_{11,5}$} & \multirow{2}{*}{$=$} & 1 & 6 & -15 & \begin{tabular}{l|l}
-4 \\
\end{tabular} & $\overline{-7}$ & $\bar{~} 10$ & 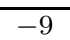 & $\overline{18}$ \\
\hline & & -2 & -14 & 3 & 8 & 17 & -11 & 12 & -13 \\
\hline \multirow{2}{*}{$V_{1}$} & \multirow{2}{*}{$=$} & 2 & 8 & 5 & -7 & -4 & $\overline{12}$ & -6 & -10 \\
\hline & & -3 & -16 & -17 & 11 & 14 & $\begin{array}{l}-13 \\
\end{array}$ & 9 & 15 \\
\hline \multirow{2}{*}{$V_{3}$} & \multirow{2}{*}{$=$} & 1 & 6 & -16 & -7 & -5 & \begin{tabular}{|c|}
-9 \\
\end{tabular} & 13 & 17 \\
\hline & & -2 & -14 & 4 & 11 & 15 & 8 & -10 & -12 \\
\hline \multirow{2}{*}{$V_{5}$} & \multirow[b]{2}{*}{$=$} & 1 & -11 & 4 & -8 & 17 & 13 & -6 & -10 \\
\hline & & -2 & 3 & -16 & 12 & -7 & -14 & 9 & 15 \\
\hline \multirow{2}{*}{$V_{7}$} & \multirow[b]{2}{*}{$=$} & 1 & -17 & -16 & -6 & 15 & 12 & 14 & -3 \\
\hline & & -2 & 9 & 4 & 10 & -5 & -13 & -11 & 8 \\
\hline \multirow{2}{*}{$V_{9}$} & \multirow{2}{*}{$=$} & 1 & -11 & 5 & 12 & -6 & 13 & -4 & -10 \\
\hline & & -2 & 3 & -17 & -8 & 16 & -14 & 7 & 15 \\
\hline \multirow{2}{*}{$V_{11}$} & & 1 & 5 & -16 & -6 & -7 & 14 & 12 & -3 \\
\hline & $=$ & -2 & -13 & 4 & 10 & 17 & -15 & -9 & 8 \\
\hline & & 1 & 9 & -15 & 16 & -4 & 6 & -8 & -5 \\
\hline$V_{13}$ & - & -2 & -17 & 3 & -12 & \begin{tabular}{|l|}
14 \\
\end{tabular} & -7 & 11 & 10 \\
\hline & & 1 & 9 & 4 & 12 & \begin{tabular}{|l|}
-3 \\
\end{tabular} & -7 & -11 & -5 \\
\hline$V_{15}$ & $=$ & -2 & -17 & -16 & -8 & 13 & 6 & 14 & 10 \\
\hline$V_{1}$ & & 1 & 7 & 4 & -6 & -3 & 11 & -5 & -9 \\
\hline$V_{17}$ & & -2 & -15 & -16 & 10 & 13 & -12 & 8 & 14 \\
\hline
\end{tabular}

FIgURE 9. Shiftable blocks of size $2 \times 8$ satisfying condition (5.1).

integer $\mathrm{H}_{1}(m-n, n ; s, k-s)$ in the proof of Proposition 4.11, we obtain a shiftable p.f. array $A_{2}$ of size $(m-n) \times n$ such that $\operatorname{supp}\left(A_{2}\right)=\widetilde{\mathcal{B}}$, rows and columns sum to zero, each row contains $s$ filled cells and each column contains $k-s$ filled cells. Let $A$ be the p.f. array of size $m \times n$ obtained taking

$$
A=\begin{array}{l|}
A_{1} \\
\hline A_{2} \\
\hline
\end{array}
$$




\begin{tabular}{|c|c|c|c|c|c|c|c|c|c|c|c|}
\hline \multirow{2}{*}{$F_{3}$} & \multirow{2}{*}{$=$} & 1 & 4 & -7 & -13 & $\begin{array}{l}-10 \\
\end{array}$ & 28 & 23 & 17 & -14 & -29 \\
\hline & & -2 & -5 & 8 & 20 & 16 & -25 & -26 & -19 & 11 & 22 \\
\hline \multirow{2}{*}{$F_{5}$} & \multirow{2}{*}{$=$} & $\overline{1}$ & -8 & 4 & -6 & 23 & 24 & -12 & 14 & -22 & -18 \\
\hline & & -2 & 7 & -3 & 13 & -17 & -21 & 9 & -16 & 19 & 11 \\
\hline \multirow{2}{*}{$W_{7,3}$} & \multirow{2}{*}{$=$} & 1 & -15 & -5 & 21 & 18 & 12 & -1 & $\overline{-6}$ & 9 & $\begin{array}{l}-22 \\
\end{array}$ \\
\hline & & -2 & 19 & 4 & -23 & -16 & -14 & 11 & 8 & -7 & 20 \\
\hline \multirow{2}{*}{$W_{7,5}$} & \multirow{2}{*}{$=$} & $\overline{1}$ & $\overline{-10}$ & 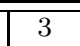 & 21 & 22 & 11 & -8 & $\overline{-7}$ & $\begin{array}{l}-15 \\
\end{array}$ & \begin{tabular}{|c|}
-18 \\
\end{tabular} \\
\hline & & -2 & 14 & -4 & -23 & -20 & -13 & 6 & 9 & 17 & 16 \\
\hline \multirow{2}{*}{$W_{7,7}$} & \multirow{2}{*}{$=$} & 1 & -19 & $\overline{-13}$ & 20 & 18 & 9 & 8 & - & $\overline{-4}$ & -17 \\
\hline & & -2 & 23 & 12 & -2 & -1 & -1 & -1 & 10 & 6 & 15 \\
\hline \multirow{2}{*}{$W_{9,5}$} & \multirow{2}{*}{$=$} & 1 & -3 & -13 & 20 & $\begin{array}{l}-4 \\
\end{array}$ & 16 & 9 & 10 & -15 & -21 \\
\hline & & -2 & 7 & 12 & -22 & 6 & -18 & -11 & -8 & 17 & 19 \\
\hline \multirow{2}{*}{$W_{9,9}$} & \multirow{2}{*}{$=$} & 1 & -7 & -17 & $\overline{-14}$ & $\overline{-13}$ & 20 & 8 & 6 & $\overline{-3}$ & 19 \\
\hline & & -2 & 11 & 16 & 12 & 15 & -22 & -10 & -4 & 5 & -21 \\
\hline \multirow{2}{*}{$W_{13,5}$} & \multirow{2}{*}{$=$} & 1 & -3 & -17 & -15 & 6 & -14 & 20 & -8 & 11 & 19 \\
\hline & & -2 & 7 & 16 & 13 & -4 & 12 & -22 & 10 & -9 & -21 \\
\hline \multirow{2}{*}{$W_{15,3}$} & \multirow{2}{*}{$=$} & 1 & -12 & 13 & 20 & 17 & -6 & 5 & -9 & -8 & -21 \\
\hline & & -2 & 16 & -14 & -2 & -1 & 15 & -7 & 11 & 10 & 19 \\
\hline \multirow{2}{*}{$W_{15,5}$} & \multirow{2}{*}{$=$} & 1 & 22 & 3 & 19 & 13 & -8 & -9 & -10 & -15 & -16 \\
\hline & & -2 & -18 & -4 & -21 & -11 & 6 & 7 & 12 & 17 & 14 \\
\hline \multirow{2}{*}{$V_{1}$} & \multirow{2}{*}{$=$} & 2 & -4 & -6 & -12 & 9 & 11 & 14 & -15 & $\overline{-18}$ & $\bar{~} 19$ \\
\hline & & -3 & 8 & 5 & 10 & -7 & -13 & -16 & 17 & 20 & -21 \\
\hline & $=$ & 1 & -9 & 11 & -7 & -4 & 8 & 14 & -15 & -18 & 19 \\
\hline$V_{3}$ & $=$ & -2 & 13 & -12 & 5 & 6 & -10 & -16 & 17 & 20 & -21 \\
\hline & & -13 & 11 & 9 & -8 & -1 & 2 & 14 & -15 & -18 & 19 \\
\hline$V_{5}$ & $=$ & 12 & -7 & -10 & 6 & 3 & -4 & -16 & 17 & 20 & -21 \\
\hline$V_{-}$ & - & 1 & 12 & 9 & -5 & -4 & -13 & 14 & -15 & -18 & 19 \\
\hline$\sqrt{7}$ & - & -2 & -8 & -10 & 3 & 6 & 11 & -16 & 17 & 20 & -21 \\
\hline$V_{0}$ & $=$ & 1 & -3 & -5 & 6 & 13 & -12 & 14 & -15 & -18 & 19 \\
\hline$\sqrt{9}$ & $=$ & -2 & 7 & 4 & -8 & -11 & 10 & -16 & 17 & 20 & -21 \\
\hline & & 1 & -9 & 7 & -6 & -3 & 10 & 14 & -15 & -18 & 19 \\
\hline$V_{11}$ & $=$ & -2 & 13 & -8 & 4 & 5 & -12 & -16 & 17 & 20 & -21 \\
\hline$V_{1}$ & $=$ & 1 & -3 & -5 & 6 & 12 & -11 & 14 & -15 & -18 & 19 \\
\hline$v_{13}$ & - & -2 & 7 & 4 & -8 & -10 & 9 & -16 & 17 & 20 & -21 \\
\hline$V_{11}$ & $=$ & 1 & -17 & 19 & 16 & 13 & 3 & $\overline{-6}$ & $\begin{array}{l}-8 \\
\end{array}$ & $\overline{-7}$ & -14 \\
\hline$V_{15}$ & $=$ & -2 & 21 & -20 & -18 & \begin{tabular}{l|l|}
8 & -1 \\
\end{tabular} & \begin{tabular}{l|l}
11 & -5 \\
\end{tabular} & 4 & 10 & 9 & 12 \\
\hline$V_{17}$ & & 1 & -3 & -5 & 6 & 12 & -11 & 13 & -14 & -18 & 19 \\
\hline$V_{17}$ & $=$ & -2 & 7 & 4 & -8 & -10 & 9 & -15 & 16 & 20 & -21 \\
\hline$V_{1}$ & & 1 & -17 & 3 & 18 & 16 & 13 & -7 & -6 & -10 & -11 \\
\hline$V_{19}$ & & -2 & 21 & -4 & -20 & -14 & \begin{tabular}{|l|l|}
4 & -15 \\
\end{tabular} & 5 & 8 & 12 & 9 \\
\hline$V_{0}$ & & 1 & -3 & -5 & 6 & 12 & -11 & 13 & -14 & -17 & 18 \\
\hline$V_{21}$ & $=$ & -2 & 7 & 4 & -8 & -10 & 9 & -15 & 16 & 19 & -20 \\
\hline
\end{tabular}

Figure 10. Shiftable blocks of size $2 \times 10$ satisfying condition (5.1). 


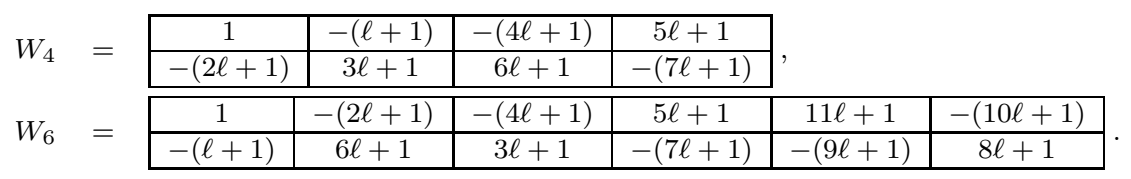

FIGURE 11. Shiftable blocks satisfying condition (5.1).

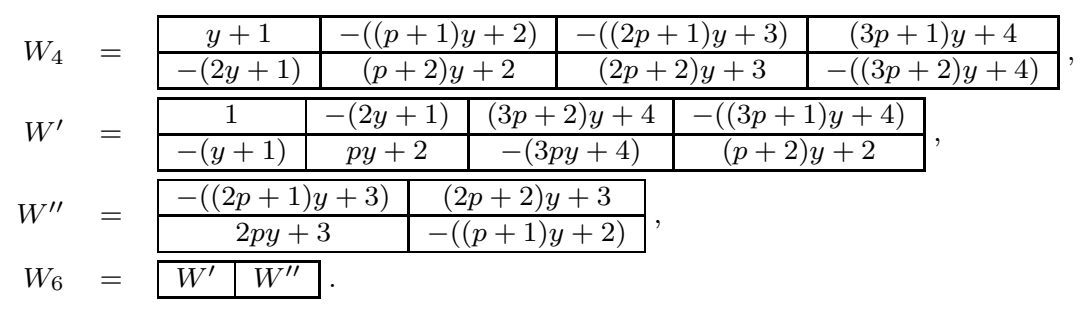

FiguRE 12. Shiftable blocks $W_{4}, W_{6}$ satisfying condition (5.1).

Each row of $A$ contains $s$ filled cells and each of its columns contains $s+(k-s)=k$ filled cells. Since $\operatorname{supp}\left(\widetilde{\mathcal{B}^{\prime}}+\widetilde{\mathcal{B}}\right)=[1, m s+\lfloor t / 2\rfloor] \backslash\{j \ell: j \in[1,\lfloor t / 2\rfloor], A$ is a shiftable integer $\mathrm{H}_{t}(m, n ; s, k)$.

An integer $\mathrm{H}_{32}(16 ; 14)$ and an integer $\mathrm{H}_{15}(20,12 ; 6,10)$ are shown in Figure 13 .

\section{Conclusions}

As the reader could observe, our constructions are all obtained taking basic blocks and arranging these blocks in some order. In all cases, changing the order of the blocks but keeping the same skeleton, one can obtain a different $\mathrm{H}_{t}(m, n ; s, k)$ for a fixed choice of $(m, n, s, k, t)$. Hence, for each case we actually produced at least $\left\lfloor\frac{m}{2}\right\rfloor$ ! different integer $\mathrm{H}_{t}(m, n ; s, k)$ with the same skeleton. Our procedures can be easily implemented in a computer: programs for GAP are available upon request writing to the second author. We also point out that the Heffter arrays $\mathrm{H}_{t}(n ; k)$ given here for $t=1,2, k$ are actually different from the arrays obtained in [3, 12].

As remarked in the introduction, (simple) relative Heffter arrays $\mathrm{H}_{t}(m, n ; s, k)$ can be used for exhibiting pairs of orthogonal cyclic decompositions of the complete multipartite graph $K_{\frac{2 m s+t}{t} \times t}$, where one decomposition consists of $s$-cycles and the other one consists of $k$-cycles. The reader interested in this type of problems can find full details in [12.

\section{REFERENCES}

[1] D.S. Archdeacon, Heffter arrays and biembedding graphs on surfaces, Electron. J. Combin. 22 (2015), \#P1.74.

[2] D.S. Archdeacon, T. Boothby and J.H. Dinitz, Tight Heffter arrays exist for all possible values, J. Combin. Des. 25 (2017), 5-35.

[3] D.S. Archdeacon, J.H. Dinitz, D.M. Donovan and E.S. Yazıcı, Square integer Heffter arrays with empty cells, Des. Codes Cryptogr. 77 (2015), 409-426.

[4] M. Buratti and A. Pasotti, On perfect $\Gamma$-decompositions of the complete graph, J. Combin. Des. 17 (2009), 197-209. 


\begin{tabular}{|c|c|c|c|c|c|c|c|c|c|c|c|c|c|c|c|}
\hline \multirow{4}{*}{$\begin{array}{l}\Omega \\
\mapsto\end{array}$} & -3 & -5 & 6 & 12 & -11 & 103 & 108 & -118 & -109 & -107 & -111 & 115 & 119 & & \\
\hline & 7 & 4 & -8 & -10 & 9 & -104 & -116 & 106 & 113 & 117 & 110 & -112 & -114 & & \\
\hline & & 13 & -21 & 23 & -19 & -16 & 20 & 121 & 129 & 124 & 132 & -123 & -127 & -131 & -125 \\
\hline & & -14 & 25 & -24 & 17 & 18 & -22 & -122 & -137 & -136 & -128 & 133 & 126 & 134 & 130 \\
\hline-145 & $\begin{array}{l}-142 \\
\end{array}$ & & & -38 & 36 & 34 & -33 & -26 & 27 & 138 & 146 & -152 & 153 & -141 & 143 \\
\hline 148 & 147 & & & 37 & -32 & -35 & 31 & 28 & -29 & -139 & -154 & 140 & -149 & 151 & -144 \\
\hline$\Xi$ & 168 & 166 & -157 & & & 39 & 50 & 47 & -43 & -42 & -51 & 155 & 159 & -170 & -160 \\
\hline 171 & $\begin{array}{l}-169 \\
\end{array}$ & -163 & 162 & & & -40 & -46 & -48 & 41 & 44 & 49 & -156 & -167 & 158 & 164 \\
\hline 176 & 183 & -177 & 184 & -175 & -181 & & & 52 & -54 & -56 & 57 & 64 & -63 & 172 & -182 \\
\hline-188 & -179 & 187 & -185 & 178 & 186 & & & -53 & 58 & 55 & -59 & -62 & 61 & -173 & 174 \\
\hline 189 & -205 & -204 & -194 & 203 & 200 & 202 & $\begin{array}{l}-191 \\
\end{array}$ & & & 65 & -73 & 71 & -70 & -67 & 74 \\
\hline-190 & 197 & 192 & 198 & -193 & -201 & -199 & 196 & & & -66 & 77 & -72 & 68 & 69 & -76 \\
\hline 89 & -88 & 206 & -216 & 209 & -213 & 222 & 218 & -211 & -215 & & & 78 & -80 & $\begin{array}{l}-82 \\
\end{array}$ & 83 \\
\hline-87 & 86 & -207 & 208 & -221 & 217 & -212 & -219 & 214 & 220 & & & -79 & 84 & 81 & -85 \\
\hline-95 & 96 & 102 & -101 & 223 & 228 & -238 & -229 & -227 & -231 & 235 & 239 & & & 91 & -93 \\
\hline 94 & -98 & -100 & 99 & -224 & -236 & 226 & 233 & 237 & 230 & -232 & -234 & & & -92 & 97 \\
\hline
\end{tabular}

\begin{tabular}{|c|c|c|c|c|c|c|c|c|c|c|c|}
\hline 1 & -3 & -5 & 6 & 12 & -11 & & & & & & \\
\hline-2 & 7 & 4 & -8 & -10 & 9 & & & & & & \\
\hline & & -25 & 23 & 21 & -20 & -13 & 14 & & & & \\
\hline & & 24 & -19 & -22 & 18 & 15 & -16 & & & & \\
\hline & & & & 26 & -28 & -30 & 31 & 38 & -37 & & \\
\hline & & & & -27 & 32 & 29 & -33 & -36 & 35 & & \\
\hline & & & & & & 39 & -41 & -43 & 44 & 50 & -49 \\
\hline & & & & & & -40 & 45 & 42 & -46 & -48 & 47 \\
\hline 63 & -62 & & & & & & & 52 & -54 & -56 & 57 \\
\hline-61 & 60 & & & & & & & -53 & 58 & 55 & -59 \\
\hline 72 & -71 & -64 & 65 & & & & & & & -76 & 74 \\
\hline-73 & 69 & 66 & -67 & & & & & & & 75 & -70 \\
\hline 77 & -78 & & & 81 & -82 & & & -86 & 88 & & \\
\hline & 90 & -91 & & & 94 & -95 & & & -98 & 100 & \\
\hline & & 103 & -104 & & & 107 & -108 & & & -111 & 113 \\
\hline-116 & & & 115 & -121 & & & 120 & 126 & & & -124 \\
\hline-79 & 80 & & & -83 & 84 & & & 87 & -89 & & \\
\hline & -92 & 93 & & & -96 & 97 & & & 99 & -101 & \\
\hline & & -105 & 106 & & & -109 & 110 & & & 112 & -114 \\
\hline 118 & & & -117 & 123 & & & -122 & -127 & & & 125 \\
\hline
\end{tabular}


[5] A. Burgess, F. Merola and T. Traetta, Cyclic cycle systems of the complete multipartite graph, J. Combin. Des. 28 (2020), 224-260.

[6] K. Burrage, D.M. Donovan, N.J. Cavenagh, and E.Ş. Yazıcı, Globally simple Heffter arrays $H(n ; k)$ when $k \equiv 0,3(\bmod 4)$, Discrete Math. $343(2020)$, \#111787.

[7] N.J. Cavenagh, J.H. Dinitz, D.M. Donovan and E.Ş. Yazıcı, The existence of square non-integer Heffter arrays, Ars Math. Contemp. 17 (2019), 369-395.

[8] N.J. Cavenagh, D. Donovan and E.Ş. Yazıcı, Biembeddings of cycle systems using integer Heffter arrays, preprint available at https://arxiv.org/abs/1906.10525.

[9] S. Costa, M. Dalai and A. Pasotti, A tour problem on a toroidal board, Austral. J. Combin., 76 (2020), 183-207.

[10] S. Costa, F. Morini, A. Pasotti and M.A. Pellegrini, A problem on partial sums in abelian groups, Discrete Math. 341 (2018), 705-712.

[11] S. Costa, F. Morini, A. Pasotti and M.A. Pellegrini, Globally simple Heffter arrays and orthogonal cyclic cycle decompositions, Austral. J. Combin. 72 (2018), 549-493.

[12] S.Costa, F. Morini, A. Pasotti and M.A. Pellegrini, A generalization of Heffter arrays, J. Combin. Des. 28 (2020), 171-206.

[13] S. Costa, A. Pasotti and M.A. Pellegrini, Relative Heffter arrays and biembeddings, to appear in Ars Math. Contemp., preprint available at https://arxiv.org/abs/1909.03064.

[14] J.H. Dinitz and A.R.W. Mattern, Biembedding Steiner triple systems and $n$-cycle systems on orientable surfaces, Austral. J. Combin. 67 (2017), 327-344.

[15] J.H. Dinitz and I.M. Wanless, The existence of square integer Heffter arrays, Ars Math. Contemp. 13 (2017), 81-93.

[16] F. Merola, A. Pasotti and M.A. Pellegrini, Cyclic and symmetric hamiltonian cycle systems of the complete multipartite graph: an even number of parts, Ars Math. Contemp. 12 (2017), 219-233.

Dipartimento di Scienze Matematiche, Fisiche e Informatiche, Universitì di Parma, Parco Area Delle Scienze 53/A, 43124 Parma, Italy

E-mail address: fiorenza.morini@unipr.it

Dipartimento di Matematica e Fisica, Università Cattolica del Sacro Cuore, Via Musei 41, 25121 Brescia, ItAly

E-mail address: marcoantonio.pellegrini@unicatt.it 